\title{
An incremental-secant mean-field homogenization method with second statistical moments for elasto-visco-plastic composite materials
}

\author{
L. Wu ${ }^{\mathrm{a}, *}$, L. Adam ${ }^{\mathrm{b}}$, I. Doghri ${ }^{\mathrm{b}, \mathrm{c}}$, L. Noels ${ }^{\mathrm{a}}$ \\ ${ }^{a}$ University of Liege, Department of Aeronautics and Mechanical Engineering - Computational 8 \\ Multiscale Mechanics of Materials \\ Allée de la découverte 9, B-4000 Liège, Belgium \\ ${ }^{b}$ e-Xstream Engineering, Rue Emile Francqui 9, B-1435 Mont-Saint-Guibert, Belgium \\ ${ }^{c}$ Université Catholique de Louvain, Institute of Mechanics, Materials and Civil Engineering, Bâtiment \\ Euler, 1348 Louvain-la-Neuve, Belgium
}

\begin{abstract}
This paper presents an extension of the recently developed incremental-secant mean-field homogenization $(\mathrm{MFH})$ procedure in the context of elasto-plasticity to elasto-visco-plastic composite materials while accounting for second statistical moments. In the incrementalsecant formulation, a virtual elastic unloading is performed at the composite level in order to evaluate the residual stress and strain states in the different phases, from which a secant MFH formulation is applied. When applying the secant MFH process, the LinearComparison-Composite is built from the piece-wise heterogeneous residual strain-stress state using naturally isotropic secant tensors defined using either first or second statistical moment values. As a result non-proportional and non-radial loading conditions can be considered because of the incremental-secant formulation, and accurate predictions can be obtained as no isotropization step is required. The limitation of the incrementalsecant formulation previously developed was the requirement in case of hard inclusions to cancel the residual stress in the matrix phase, resulting from the composite material unloading, to avoid over-stiff predictions. It is shown in this paper that in the case of hard inclusions by defining a proper second statistical moment estimate of the von Mises stress, the residual stress can be kept in the different composite phases. Moreover it is shown that the method can be extended to visco-plastic behaviors without modifying the homogenization process as the incremental-secant formulation only requires the definition of the secant operator of the different phase material models. Finally, it is shown that although it is also possible to define a proper second statistical moment estimate of the von Mises stress in the case of soft inclusions, this does not improve the accuracy as compared to the increment-secant method with first order statistical moment estimates.
\end{abstract}

Keywords: Mean-Field Homogenization, Composites, Elasto-visco-plasticity, Incremental-secant, Second statistical moments

*Corresponding author, Phone: +32 436694 53, Fax: +32 43669505

Email address: L.Wu@ulg.ac.be, (L. Wu )

Preprint submitted to Elsevier 


\section{Introduction}

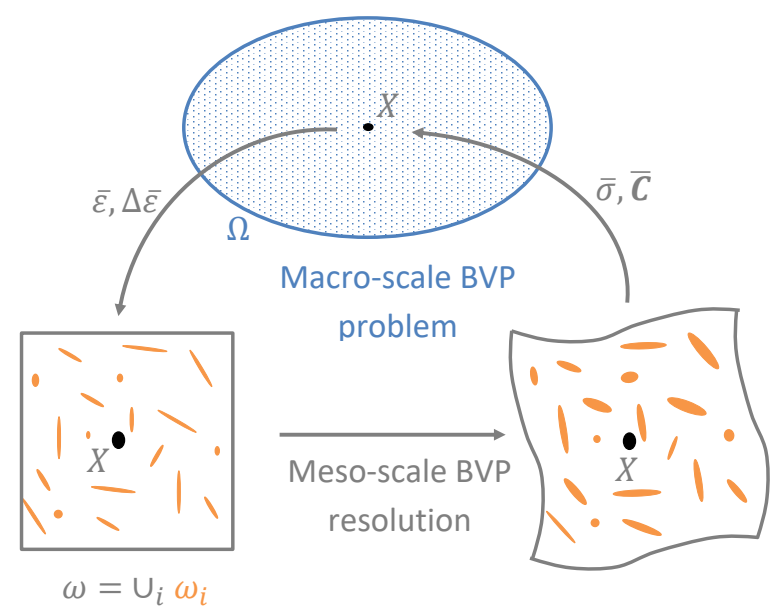

Figure 1: Homogenization-based multi-scale method

As the use of engineered and/or heterogeneous materials is gaining popularity, structural numerical simulations ought to account for the micro-scale behavior of those materials to remain accurate. As direct numerical simulations accounting explicitly for this material structure cannot reasonably be envisioned due to the overwhelming computational cost involved, computationally efficient and accurate multi-scale methods become a requirement. Among the existing multi-scale methods, homogenization-based ones are extensively studied. In such an approach, the macro-scale structure $\Omega$ defines a boundary-value-problem (BVP) which is solved by considering homogenized material properties extracted, at each (macro) material point $\boldsymbol{X}$ of interest, from the resolution of a meso-scale BVP, see Fig. 1. This meso-scale BVP is defined on a meso-scale volume $\omega$ which represents the different phases $\omega_{i}$ of the material. In case of statistical representativity of the meso-scale problem, the latter is referred to as Representative Volume Element (RVE). The resolution of this meso-scale BVP links the macro-, or homogenized, stress tensor $\overline{\boldsymbol{\sigma}}$ and the macro-, or homogenized-, strain tensor $\overline{\boldsymbol{\varepsilon}}$, which correspond to the average values of respectively the local stress tensor $\boldsymbol{\sigma}$ and strain tensor $\boldsymbol{\varepsilon}$ on the RVE $\omega$. In case of non-linear behaviors, the increment of the macro-, or homogenized-, strain tensor $\Delta \bar{\varepsilon}$ is also required. Besides, the homogenized material tensor $\bar{C}$ also arises from the meso-scale BVP resolution.

Several homogenization techniques were developed, see the reviews by Kanouté et al. (2009); Geers et al. (2010); LLorca et al. (2011); Nemat-Nasser and Hori (2013); Noels et al. (2016). The methods can be categorized into purely numerical ones and semianalytical ones. Among the former ones, the Voronoï cell finite element method (VCFEM) developed by Ghosh et al. (1995) divides the macro-scale problem into Voronoï cells considered as germination points of the heterogeneities. This method was generalized by Michel et al. (1999); Feyel (1999); Terada et al. (2000); Miehe (2002); Kouznetsova et al. 
(2001) by associating to each macro-scale integration point a finite element model of the RVE, leading to the so-called computational homogenization or $\mathrm{FE}^{2}$ method. To avoid the difficulty of meshing the RVE, the Fast-Fourier-Transforms (FFT) method, in which the RVE is discretized as data sampled from a regular grid, was developed by Moulinec and Suquet (1995, 1998); Michel et al. (2001). Although such numerical homogenization resolutions can handle general heterogeneous micro-structures and general classes of non-linear models of the micro-constituent material behaviors, the computational cost can be prohibitive particularly for 3D applications. Therefore semi-analytical homogenization methods remain attractive. Among them, one can find the Generalized Method of Cells (GMC) discretizing the RVE by parallelepiped sub-cells and reviewed by Aboudi (1996), the Transformation-Field Analysis (TFA), which divides the RVE into sub-domains having at most one phase and associated to an eigen-strain tensor representative of the non-linear behavior, as pioneered by Dvorak et al. (1994), or its extension called non-Uniform Transformation-Field Analysis (NTFA) developed by Michel and Suquet (2003) in order to avoid the sub-division of one phase into sub-domains. Finally the Mean-Field Homogenization (MFH) considers as homogenized strain and stress fields the volume average of their respective counterparts in the different composite material phases, while the relation between the average strain tensors in the different phases is obtained through the definition of an ad-hoc strain concentration tensor. This strain concentration tensor is usually obtained as an extension of the single inclusion solution of Eshelby (1957) to multiple inclusions interacting in an average way in the composite material, usually either through the Mori and Tanaka (1973) method or through the self-consistent scheme as developed by Kröner (1958); Hill (1965b).

Being computationally efficient, MFH methods were extensively developed to account for non-linear material behaviors. In such a case, the linear equations are applied on a so-called linear comparison composite (LCC) (Talbot and Willis, 1985, 1987; Ponte Castañeda, 1991, 1992; Talbot and Willis, 1992; Molinari et al., 2004). This LCC is defined as a virtual composite material whose constituents linear behaviors behave similarly to the linearized behaviors of the real constituents at given strain states. The definition of the LCC in terms of the non-linear behavior that is ought to be captured is the key ingredient of the MFH methods in order to obtain accurate predictions. In the secant method developed by Berveiller and Zaoui (1978) for elasto-plastic (EP) materials, the LCC is defined by joining the origin to the current strain-stress state. However, in this case the linearized law is pseudo-elastic in terms of the total stress and strain tensors, which limits the applicability of the method to monotonic and proportional loading conditions. To circumvent this limitation, the incremental-tangent formulation was developed by Hill (1965a); Pettermann et al. (1999); Doghri and Ouaar (2003); Doghri and Tinel (2005) for EP behaviors. Such an incremental-tangent MFH approach was combined with (macro)-localization enhancements and phenomenological fracture models by Ostlund et al. (2016) and was extended by Wu et al. (2012) to account for non-local (micro) damage enhanced EP behaviors. In the incremental-tangent formulation, the LCC is defined from a linearized relation between the stress and strain increments of the different constituents around their current strain-stress states. In order to model elasto-visco-plastic (EVP) behaviors, the affine method, in which the total strain field in combination with a stress polarization tensor is considered instead of the strain increments to define the LCC, was developed by Molinari et al. (1987); Masson and Zaoui (1999); Masson et al. (2000); Molinari et al. (2004); Pierard and Doghri (2006a); Pier- 
ard et al. (2007); Mercier and Molinari (2009); Doghri et al. (2010). Although both affine and incremental-tangent formulations can handle non-monotonic loading conditions, their prediction is over-stiff unless the matrix tangent-operator is "isotropized" during the homogenization process as shown by Doghri and Ouaar (2003); Chaboche et al. (2005); Pierard et al. (2007). To avoid the isotropization step, Wu et al. (2013a) and Wu et al. (2013b) -the authors- have developed an incremental-secant approach for respectively EP and non-local-damage-enhanced EP behaviors. In this approach, the homogenized material is virtually unloaded and a secant approach is then applied from the residual strains obtained in the different phases. On the one hand, this method does not require an isotropization step as the LCC is naturally defined by isotropic instantaneous stiffness tensors, and on the other hand the method handles non-monotonic and nonproportional loading conditions. The limitation of the incremental-secant formulation in case of hard inclusions is the requirement to cancel the residual stress in the matrix phase, obtained after total material unloading, to avoid over-stiff predictions.

Most of the cited references for MFH methods considers the first statistical moment values in the different phases when predicting their plastic flow. As a result the plastic yield is not always accurately captured in some material systems such as short fibers composite materials, as discussed by Moulinec and Suquet (2003), which could result in inaccurate predictions. Second statistical moment values were thus accounted for in the flow rule computation of the different phases for EP composite materials in the works of Suquet (1995) using the so-called modified-secant approach, in the work of Ponte Castañeda (1996); Doghri et al. (2011) using the incremental tangent method, and in the work of $\mathrm{Wu}$ et al. (2015a) using the incremental-secant method. In order to capture with more accuracy the field fluctuations, Ponte Castañeda (2002a,b) have considered "generalized secant moduli" incorporating both first and second statistical moment information and have applied the method to visco-plastic composite materials.

$\mathrm{MFH}$ methods were also extensively developed based on variational principles. Suquet (1995) has demonstrated that the variational homogenization form pioneered by Ponte Castañeda (1992) is equivalent to a second-order secant-formulation, which was renamed modified-secant approach. In order to be able to account for non-monotonic loading, incremental variational formulations, which can also include second statistical moment estimates, were developed by Lahellec and Suquet $(2007 \mathrm{a}, \mathrm{b})$ in the context of inelasticity, by Lahellec et al. (2011) in the context of thermo-elasticity, and by Brassart et al. (2011, 2012); Lahellec and Suquet (2013); Boudet et al. (2016) in the context of elasto-(visco-)plasticity. In particular the formulation developed by Lahellec and Suquet (2013) is able to handle non-radial loading conditions. The difficulty in the variational approach lies in the definition of the LCC, which is used to define the elastic and dissipative potentials. The LCC are defined in the different phases from a polarization strain and from linear properties, which are obtained from an optimization process. The optimization process ensures that the potential values are as close as possible to the energy of the non-uniform stress-strain states. In the method developed by Brassart et al. (2011, 2012), the polarization was uniform in the composite material yielding a time-step dependency for perfectly plastic behaviors; in the method developed by Lahellec et al. (2011); Lahellec and Suquet (2013) the polarization tensor and the linear properties are piece-wise-heterogeneous; and in the more recent work of Idiart and Lahellec (2016) they are point-wise-heterogeneous, improving the predictions for solids with strongly fluctuating polarization fields. Finally, in the work of Boudet et al. (2016), isotropic and 
kinematic hardening are accounted for in an accurate way by appropriate linearizations of both the stored-energy function and the dissipative potential. For completeness, we note that Ponte Castañeda (2012); Agoras and Ponte Castañeda (2013) have developed the iterated variational homogenization, in which the micro-structure is iteratively and incrementally constructed by the addition of the matrix phase to improve the estimations for non-linear composite materials.

One advantage of the incremental-secant method developed by Wu et al. (2013a, 2015a) and Wu et al. (2013b, 2015b) for respectively EP and non-local-damage-enhanced EP behaviors is its straightforward implementation as the homogenization directly calls the material constitutive material boxes of an existing materials library for the different phases. In this approach, upon the virtual unloading residual strains are obtained in the different phases corresponding to piece-wise heterogeneous residual stress tensors, and ensuring the time-increment objectivity of the method. Moreover because the LCC is defined from a virtually unloaded composite material state, on the one hand the method can handle non-proportional and non-radial loading conditions, and on the other hand LCC material tensors are naturally isotropic avoiding the isotropization step required by both the affine and incremental-tangent approaches. The limitation of the method for material systems with hard inclusions lies in the requirement to cancel the residual stress in the matrix phase (but not the residual strain neither the residual values in the inclusion phase) before applying the secant homogenization. This need to consider the so-called zero-incremental-secant method was observed when accounting for only first statistical moment (Wu et al., 2013a) but also when accounting for second statistical moments with the second statistical moment estimate of the von Mises stress proposed by $\mathrm{Wu}$ et al. (2015a). In this work we use a new second statistical moment estimate of the von Mises stress, yielding accurate results while keeping the residual stress in the matrix phase, i.e. when using the so-called residual-incremental-secant method. Moreover, because of the existing advantages of the method, there is an interest in extending it to account for non-linear EVP behaviors while considering second statistical moment estimates, which is the aim of this paper. In particular it is shown that the basis of the homogenization process of the incremental-secant method does not require to be changed because of the visco-plastic behaviors, and that all the modifications are limited to the constitutive material boxes of the material library, which makes the method particularly attractive for numerical applications.

The organization of the work is as follows. In Section 2 generalities on MFH are given, with a particular emphasis on the definition of the LCC using first and second statistical moment estimates. The key ideas of the incremental-secant method previously developed for EP composite materials are summarized. In Section 3, the Perzyna's constitutive EVP model is described, first at the micro-structural material point level, then when performing a volume average on a given material phase while accounting for the second statistical moment estimate of the von Mises stress. The resolution of the volume averaged equations, still in a given material phase, is then detailed in the context of an incremental-secant approach. The implementation of the incremental-secant MFH method is then summarized in Section 4. In particular, it is shown that the MFH scheme is not modified by the viscous term in the phase constitutive behavior. Finally, the method predictions are compared to finite element (FE) results, to Fast-FourierTransform (FFT) results, to experiments and to other homogenization predictions in Section 5. In particular non-proportional loading conditions are considered to demon- 
strate the objectivity of the method.

\section{Mean-field homogenization and incremental-secant approach}

In this section, the definition of a LCC is discussed in a general way in the context of the MFH of non-linear materials. In particular, the first and second statistical moment estimates are defined. Then the definition of the LCC is particularized to the incremental secant approach.

\subsection{Generalities on the mean-field homogenization for two-phase composites}

In a multiscale approach, each macro-point $\boldsymbol{X}$ of the structure is viewed as the center of a RVE of domain $\omega$ at the micro-level, see Fig. 1. The Hill-Mandel condition, which states the energy equivalence at the two scales, implies that the relation between the macro-strain tensor $\bar{\varepsilon}$ and macro-stress tensor $\overline{\boldsymbol{\sigma}}$ is equivalent to the relation between the volume averages of the micro-strain tensor $\langle\varepsilon\rangle_{\omega}$ and micro-stress tensor $\langle\boldsymbol{\sigma}\rangle_{\omega}$ over the RVE. Considering a two-phase composite material with the respective volume fractions $v_{0}+v_{\mathrm{I}}=1$ (subscript 0 refers to the matrix and I to the inclusions), the volume average over the RVE $\omega$ can be explicitly expressed in terms of the volume averages over the two phases $\omega_{0}$ and $\omega_{\mathrm{I}}$, with

$$
\overline{\boldsymbol{\varepsilon}}=v_{0}\langle\boldsymbol{\varepsilon}\rangle_{\omega_{0}}+v_{\mathrm{I}}\langle\boldsymbol{\varepsilon}\rangle_{\omega_{\mathrm{I}}} \quad \text { and } \quad \overline{\boldsymbol{\sigma}}=v_{0}\langle\boldsymbol{\sigma}\rangle_{\omega_{0}}+v_{\mathrm{I}}\langle\boldsymbol{\sigma}\rangle_{\omega_{\mathrm{I}}} .
$$

The key-point of the MFH method is to define the relation between the strain averages in the different phases, using a strain concentration tensor $\mathbb{B}^{\epsilon}$. For linear material systems, this implies

$$
\bar{\varepsilon}_{\mathrm{I}}=\mathbb{B}^{\epsilon}\left(\mathrm{I}, \mathbb{C}_{0}^{\mathrm{el}}, \mathbb{C}_{\mathrm{I}}^{\mathrm{el}}\right): \bar{\varepsilon}_{0},
$$

where $\mathbb{C}_{i}^{\text {el }}$ is the elastic stiffness tensor in phase $\omega_{i}$, I represents the geometry of the inclusion, and where we have used $\boldsymbol{\bullet}_{i}$ to represent the volume average over the phase $\omega_{i}$, i.e. $\langle\bullet\rangle_{\omega_{i}}$, for conciseness. Note that $\left\langle\boldsymbol{\bullet}_{\omega_{i}}=\overline{\boldsymbol{\bullet}}_{i}\right.$ represents the first statistical value estimate of the field $\bullet$. A popular approach to define the strain concentration tensor $\mathbb{B}^{\epsilon}$ is to extend the single inclusion solution of Eshelby (1957) to multiple inclusions interacting in an average way. In particular, in this paper we consider the Mori and Tanaka (1973) method (M-T), in which the average strain in the matrix phase of the multiple inclusions composite material corresponds to the strain at infinity of the single inclusion problem, with

$$
\mathbb{B}^{\epsilon}\left(\mathrm{I}, \mathbb{C}_{0}^{\mathrm{el}}, \mathbb{C}_{\mathrm{I}}^{\mathrm{el}}\right)=\left\{\mathbb{I}+\mathbb{S}:\left[\left(\mathbb{C}_{0}^{\mathrm{el}}\right)^{-1}: \mathbb{C}_{\mathrm{I}}^{\mathrm{el}}-\mathbb{I}\right]\right\}^{-1},
$$

where the Eshelby tensor (Eshelby, 1957) $\mathbb{S}\left(\mathrm{I}, \mathbb{C}_{0}^{\mathrm{el}}\right)$ depends on the geometry of the inclusion (I) and on the elastic tensor of the matrix phase $\mathbb{C}_{0}^{\mathrm{el}}$.

For non-linear material systems, the strain concentration tensor is constructed using a linear comparison composite (LCC), yet to be defined, which represents the linearized behavior of the composite material phases through their virtual elastic operators, i.e. $\hat{\mathbb{C}}_{0}^{\mathrm{LCC}}$ for the matrix phase and $\hat{\mathbb{C}}_{I}^{\mathrm{LCC}}$ for the inclusions phase. Eq. (2) is thus rewritten

$$
\Delta \bar{\varepsilon}_{\mathrm{I}}=\mathbb{B}^{\epsilon}\left(\mathrm{I}, \hat{\mathbb{C}}_{0}^{\mathrm{LCC}}, \hat{\mathbb{C}}_{\mathrm{I}}^{\mathrm{LCC}}\right): \Delta \bar{\varepsilon}_{0} .
$$

Depending on the assumptions behind the definition of the LCC, the tensors $\hat{\mathbb{C}}_{0}^{\mathrm{LCC}}$ and $\hat{\mathbb{C}}_{I}^{\mathrm{LCC}}$ are constructed in different ways. However, they are constructed in such a way 
that they are uniform over each phase, hence the $\hat{\bullet}$ notation. In the secant method developed by Berveiller and Zaoui (1978) the material is linearized as $\overline{\boldsymbol{\sigma}}=\hat{\mathbb{C}}^{\mathrm{sec}}: \bar{\varepsilon}$, in which case only monotonic loading conditions can be considered. In the incrementaltangent formulation pioneered by Hill (1965a); Pettermann et al. (1999), the non-linear material is linearized as $d \overline{\boldsymbol{\sigma}}=\hat{\mathbb{C}}^{\text {tan }}: d \overline{\boldsymbol{\varepsilon}}$, where $\hat{\mathbb{C}}^{\text {tan }}$ is an EP tangent operator, allowing to consider unloading. In the affine method, which was first proposed by Molinari et al. (1987, 2004) for visco-plastic materials, the material is linearized using a polarization stress $\boldsymbol{\tau}$ as $\overline{\boldsymbol{\sigma}}=\hat{\mathbb{C}}: \overline{\boldsymbol{\varepsilon}}+\boldsymbol{\tau}$, where $\hat{\mathbb{C}}$ can be different from the tangent moduli.

In order to improve the predictions, MFH was enriched by accounting for the second statistical moment estimates $\langle\bullet \otimes \bullet\rangle_{\omega_{i}}$ of field $\bullet$. In particular, the second statistical moment estimate of the equivalent strain increment in phase $\omega_{i}$ reads

$$
\Delta \hat{\hat{\varepsilon}}_{i}^{\mathrm{eq}}=\sqrt{\frac{2}{3} \mathbb{I}^{\mathrm{dev}}::\langle\Delta \varepsilon \otimes \Delta \varepsilon\rangle_{\omega_{i}}},
$$

and the second statistical moment estimate of the equivalent stress increment in the phase $\omega_{i}$ reads

$$
\Delta \hat{\hat{\sigma}}_{i}^{\mathrm{eq}}=\sqrt{\frac{3}{2} \mathbb{I}^{\mathrm{dev}}::\langle\Delta \boldsymbol{\sigma} \otimes \Delta \boldsymbol{\sigma}\rangle_{\omega_{i}}},
$$

where $\mathbb{I}^{\text {dev }}$ is the deviatoric fourth-order tensor. In particular, the second statistical moment estimate of the incremental strain field in the phase $\omega_{i}$ can be computed by

$$
\langle\Delta \varepsilon \otimes \Delta \varepsilon\rangle_{\omega_{i}}=\frac{1}{v_{i}} \Delta \bar{\varepsilon}: \frac{\partial \hat{\mathbb{C}}^{\mathrm{LCC}}}{\partial \hat{\mathbb{C}}_{i}^{\mathrm{LCC}}}: \Delta \bar{\varepsilon},
$$

as detailed by Bobeth and Diener (1987); Buryachenko (2001), where $\Delta \bar{\varepsilon}=\langle\Delta \varepsilon\rangle_{\omega}$ and where $\hat{\mathbb{C}}^{\mathrm{LCC}}$ is the effective elastic tensor of the LCC, corresponding to the given phase virtual elastic operators $\hat{\mathbb{C}}_{0}^{\mathrm{LCC}}$ and $\hat{\mathbb{C}}_{\mathrm{I}}^{\mathrm{LCC}}$.

\subsection{Incremental-secant approach}

Recently, Wu et al. (2013a, 2015a), the authors, have defined the LCC using an incremental-secant method which is able to deal with non-proportional loading conditions of EP materials. In such an approach each phase of the material is linearized as $\overline{\boldsymbol{\sigma}}_{i}=\hat{\mathbb{C}}_{i}^{\mathrm{S}}$ : $\Delta \bar{\varepsilon}_{i}^{\mathrm{r}}+\overline{\boldsymbol{\sigma}}_{i}^{\text {res }}$, where $\overline{\boldsymbol{\sigma}}_{i}^{\text {res }}$ is obtained from a virtual unloading of the composite material, where $\Delta \bar{\varepsilon}_{i}^{\mathrm{r}}$ is the strain increment from the virtual unloaded state, and where $\hat{\mathbb{C}}_{0}^{\mathrm{S}}$ and $\hat{\mathbb{C}}_{\mathrm{I}}^{\mathrm{S}}$ are the secant operators defined from a virtual unloaded state. As the operator $\hat{\mathbb{C}}_{0}^{S}$ and $\hat{\mathbb{C}}_{\mathrm{I}}^{\mathrm{S}}$ are naturally isotropic for $\mathrm{J}_{2}$-EP materials, the isotropization step required with the incremental-tangent and incrementally affine methods (Chaboche et al., 2005; Pierard and Doghri, 2006b; Doghri et al., 2010) is avoided.

Considering first the material level at position $\boldsymbol{x} \in \omega$, i.e. without homogenizing the material, in an incremental process of a non-linear material, we consider that the total strain tensor $\varepsilon_{n}$, the stress tensor $\sigma_{n}$, and the internal variables are known at time $t_{n}$, the beginning of a time interval $\left[t_{n}, t_{n+1}\right]$. During that step, the increment of the stress tensor is computed for a given strain increment $\Delta \varepsilon_{n+1}$, see Fig. 2. The material state at time $t_{n+1}$ thus reads

$$
\begin{aligned}
& \varepsilon_{n+1}(\boldsymbol{x})=\varepsilon_{n}(\boldsymbol{x})+\Delta \varepsilon_{n+1}(\boldsymbol{x}) \quad \text { and } \\
& \boldsymbol{\sigma}_{n+1}(\boldsymbol{x})=\boldsymbol{\sigma}_{n}(\boldsymbol{x})+\Delta \boldsymbol{\sigma}_{n+1}(\boldsymbol{x}) \quad \forall \boldsymbol{x} \in \omega_{i} . \\
& 7
\end{aligned}
$$




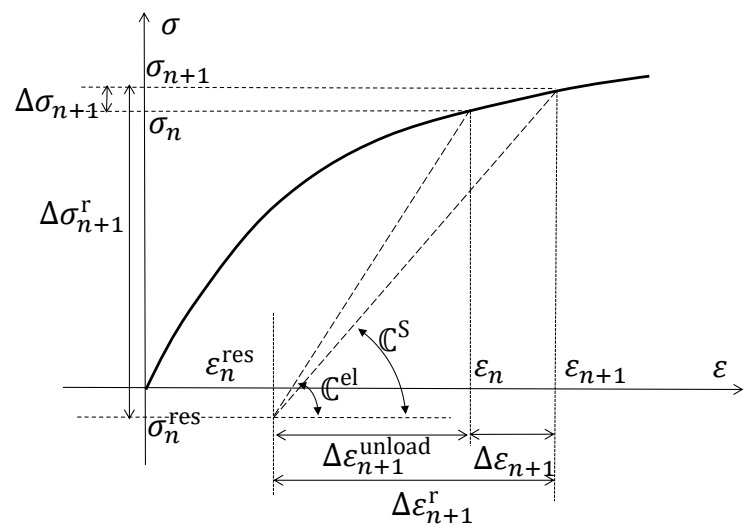

Figure 2: Schematics of the incremental-secant formulation in each phase, from Wu et al. (2013a). The incremental-secant operator is defined from the residual strain and stress.

In the incremental-secant framework, a virtual elastic unloading step from the stress state $\boldsymbol{\sigma}_{n}$ is applied at time $t_{n}$, which defines a residual strain tensor $\varepsilon_{n}^{\text {res }}$, see Fig. 2. Eqs. (8) are then rewritten as

$$
\begin{array}{ll}
\varepsilon_{n+1}(\boldsymbol{x})=\varepsilon_{n}^{\mathrm{res}}(\boldsymbol{x})+\Delta \varepsilon_{n+1}^{\mathrm{r}}(\boldsymbol{x}) & , \text { and } \\
\boldsymbol{\sigma}_{n+1}(\boldsymbol{x})=\boldsymbol{\sigma}_{n}^{\text {res }}(\boldsymbol{x})+\Delta \boldsymbol{\sigma}_{n+1}^{\mathrm{r}}(\boldsymbol{x}) & \forall \boldsymbol{x} \in \omega_{i} .
\end{array}
$$

Now considering the homogenized material, in the incremental-secant MFH framework, the LCC is defined so that the composite material is subjected to a homogenized strain increment $\Delta \bar{\varepsilon}_{n+1}^{\mathrm{r}}$, satisfying

$$
\Delta \overline{\boldsymbol{\sigma}}_{n+1}^{\mathrm{r}}=\hat{\mathbb{C}}^{\mathrm{S}}: \Delta \bar{\varepsilon}_{n+1}^{\mathrm{r}},
$$

which corresponds for each phase $\omega_{i}$ to

$$
\Delta \overline{\boldsymbol{\sigma}}_{i n+1}^{\mathrm{r}}=\hat{\mathbb{C}}_{i}^{\mathrm{S}}: \Delta \bar{\varepsilon}_{i n+1}^{\mathrm{r}} .
$$

Correspondingly, relation (4) now reads

$$
\Delta \bar{\varepsilon}_{\mathrm{I}}^{\mathrm{r}}=\mathbb{B}^{\epsilon}\left(\mathrm{I}, \hat{\mathbb{C}}_{0}^{\mathrm{S}}, \hat{\mathbb{C}}_{\mathrm{I}}^{\mathrm{S}}\right): \Delta \bar{\varepsilon}_{0}^{\mathrm{r}} .
$$

Finally, the phase volume averaged stress and strain states follow from (9), with

$$
\begin{aligned}
& \bar{\varepsilon}_{i n+1}=\bar{\varepsilon}_{i n}^{\text {res }}+\Delta \bar{\varepsilon}_{i n+1}^{\mathrm{r}}, \text { and } \\
& \overline{\boldsymbol{\sigma}}_{i n+1}=\overline{\boldsymbol{\sigma}}_{i n}^{\mathrm{res}}+\Delta \overline{\boldsymbol{\sigma}}_{i n+1}^{\mathrm{r}} .
\end{aligned}
$$

The incremental secant approach has been developed to predict the macro-behaviors of composite materials with EP phases by considering the first statistical moment estimates by $\mathrm{Wu}$ et al. (2013a). Moreover the case of damage-enhanced EP matrix was also considered in Wu et al. (2013b, 2015b). Finally the method has been extended to account for the second statistical moment estimates in EP phases by Wu et al. (2015a). The purpose of this paper is to extend the method to be able to account for elasto-visco-plastic material phases. 


\section{3. $\mathbf{J}_{2}$-plasticity formulation of elasto-visco-plastic materials with second sta- tistical moment estimates}

In this section we first consider the local, i.e. at the micro-material point level, $\mathrm{J}_{2}$-EVP equations based on a Perzyna's constitutive model. The volume averaged constitutive equations, in a given material phase $\omega_{i}$, are then described when accounting for the second statistical moment estimate of the von Mises stress. The predictor-corrector and return mapping schemes associated to these volume averaged constitutive equations are then detailed. Finally, the incremental-secant operators corresponding to the incremental-secant formalism described in Section 2.2 are then evaluated from the volume averaged constitutive equations.

\section{1. $J_{2}$-elasto-visco-plasticity with Perzyna's formulation}

In this section, we recall the basic formulations of the Perzyna's visco-plastic model for homogeneous materials. The total strain tensor $\varepsilon$ is decomposed into an elastic part and a visco-plastic part,

$$
\varepsilon=\varepsilon^{\mathrm{el}}+\varepsilon^{\mathrm{vp}}, \quad \text { and } \quad \boldsymbol{\sigma}=\mathbb{C}^{\mathrm{el}}:\left(\varepsilon-\varepsilon^{\mathrm{vp}}\right),
$$

where $\boldsymbol{\sigma}$ is the Cauchy stress tensor and $\mathbb{C}^{\mathrm{el}}$ is the elastic (Hooke's) material tensor.

The visco-plastic strain tensor is computed from a potential $\phi\left(\sigma^{\mathrm{eq}}, p\right)$ with

$$
\dot{\boldsymbol{\varepsilon}}^{\mathrm{vp}}=\left\{\begin{array}{ll}
\frac{\partial \phi}{\partial \boldsymbol{\sigma}}=\frac{3}{2} \frac{\boldsymbol{\sigma}^{\mathrm{dev}}}{\sigma^{\mathrm{eq}}} \frac{\partial \phi}{\partial \sigma^{\mathrm{eq}}} & \text { if } f>0 \\
0 & \text { if } f \leq 0
\end{array},\right.
$$

and

$$
\dot{\gamma}=\left\{\begin{array}{ll}
\frac{\partial \phi}{\partial \sigma^{\mathrm{eq}}}=g_{\mathrm{v}}\left(\sigma^{\mathrm{eq}}, p\right)>0 & \text { if } f>0 \\
0 & \text { if } f \leq 0
\end{array},\right.
$$

where $\sigma^{\text {eq }}$ is the von Mises equivalent stress, $\sigma^{\text {dev }}=\mathbb{I}^{\text {dev }}: \sigma$ is the deviatoric part of the stress tensor, $\dot{\gamma}$ is the visco-plastic multiplier rate, and $g_{\mathrm{v}}$ is a visco-plastic function. In these two equations, we have use $f$ the yielding function

$$
f=\sigma^{\mathrm{eq}}-\sigma_{\mathrm{Y}}-R(p)
$$

where $\sigma_{\mathrm{Y}}$ is the initial yielding stress, $R(p)$ is an isotropic hardening stress, and $p$ is the accumulated plastic strain. Because the potential function $\phi$ is based on the von Mises stress, combining Eqs. (15) and (18) leads to

$$
\dot{\gamma}=\dot{p}=\left(\frac{2}{3} \dot{\varepsilon}^{\mathrm{vp}}: \dot{\varepsilon}^{\mathrm{vp}}\right)^{\frac{1}{2}}
$$

In the time interval $\left[t_{n}, t_{n+1}\right]$, using equation (18), the discretized form of equation (16) is obtained through a fully implicit backward Euler time integration, which reads

$$
\Delta p=g_{\mathrm{v}}\left(\sigma^{\mathrm{eq}}, p\right) \Delta t,
$$

where $\Delta t=t_{n+1}-t_{n}$. 


\subsection{Elasto-visco-plastic description of volume averaged material}

In this section, the Perzyna's visco-plastic description is reformulated in each phase $\omega_{i}$ based on a second statistical moment estimate of the stress field. Since we consider only one phase which is noted as $\omega_{i}$, here $\bar{\bullet}$ is used to indicate $\langle\boldsymbol{\bullet}(\boldsymbol{x})\rangle_{\omega_{i}}$ for simplicity and without introducing any ambiguity.

A volume average on the phase $\omega_{i}$ is performed on Eq. (14), resulting into

$$
\bar{\varepsilon}=\bar{\varepsilon}^{\mathrm{el}}+\bar{\varepsilon}^{\mathrm{vp}}, \quad \text { and } \quad \overline{\boldsymbol{\sigma}}=\mathbb{C}^{\mathrm{el}}:\left(\bar{\varepsilon}-\bar{\varepsilon}^{\mathrm{vp}}\right) .
$$

To evaluate the yielding function (17), the second statistical moment estimate of the von Mises stress, i.e.

$$
\hat{\hat{\sigma}}^{\mathrm{eq}}=\sqrt{\frac{3}{2} \mathbb{I}^{\mathrm{dev}}::\langle\boldsymbol{\sigma}(\boldsymbol{x}) \otimes \boldsymbol{\sigma}(\boldsymbol{x})\rangle_{\omega_{i}}},
$$

is considered, instead of a direct volume average on phase $\omega_{i}$, leading to

$$
f=\hat{\hat{\sigma}}^{\mathrm{eq}}-R(\hat{p})-\sigma_{Y}
$$

where $\hat{p}$ is an accumulated equivalent visco-plastic strain in the sense of average, since it is computed based on $\hat{\hat{\sigma}}^{\text {eq. }}$. However, it is neither the first statistical moment nor the second statistical moment estimate of $p(\boldsymbol{x}), \forall \boldsymbol{x} \in \omega_{i}$. Therefore, the local EVP Eqs. (15)-(19) cannot be used directly to describe the response of the volume averaged phase. In order to formulate a comparable visco-plastic model for the volume averaged material, we assume that the visco-plastic multiplier rate, $\dot{\hat{\gamma}}$, of the volume averaged phase reads

$$
\left\{\begin{array}{ll}
\dot{\hat{\gamma}}=g_{\mathrm{v}}\left(\hat{\hat{\sigma}}^{\mathrm{eq}}, \hat{p}\right)>0 & \text { if } f>0 \\
\dot{\hat{\gamma}}=0 & \text { if } f \leq 0
\end{array}, \text { and } \dot{\hat{p}}=\dot{\hat{\gamma}}\right.
$$

where the visco-plastic function $g_{\mathrm{v}}$ is evaluated through $\hat{\hat{\sigma}}^{\mathrm{eq}}$ and $\hat{p}$.

Considering the time increment $\Delta t$ in a time interval $\left[t_{n}, t_{n+1}\right]$, we directly have

$$
\hat{p}=\hat{p}_{n}+\Delta \hat{p}, \text { with } \Delta \hat{p}=g_{\mathrm{v}}\left(\hat{\hat{\sigma}}^{\mathrm{eq}}, \hat{p}\right) \Delta t,
$$

which results from the fully implicit backward Euler time integration.

In order to evaluate the volume averaged visco-plastic strain, we assume

$$
\dot{\bar{\varepsilon}}^{\mathrm{vp}}=\dot{\hat{\gamma}} \hat{\hat{N}}
$$

where $\hat{\hat{\boldsymbol{N}}}$ will be defined later and is such that $\hat{\hat{\boldsymbol{N}}} \neq \frac{\partial f}{\partial \overline{\boldsymbol{\sigma}}}=\frac{\partial \hat{\hat{\hat{\sigma}}}^{\mathrm{eq}}}{\partial \bar{\sigma}^{\mathrm{eq}}} \overline{\boldsymbol{N}}$ with $\bar{\sigma}^{\mathrm{eq}}=\sqrt{\frac{3}{2} \overline{\boldsymbol{\sigma}}^{\mathrm{dev}}: \overline{\boldsymbol{\sigma}}^{\mathrm{dev}}}$ the equivalent von Mises stress of $\overline{\boldsymbol{\sigma}}$, and $\overline{\boldsymbol{N}}=\frac{\partial \bar{\sigma}^{\mathrm{eq}}}{\partial \overline{\boldsymbol{\sigma}}}=\frac{3}{2} \frac{\overline{\boldsymbol{\sigma}}^{\mathrm{dev}}}{\bar{\sigma}^{\mathrm{eq}}}$. This means that

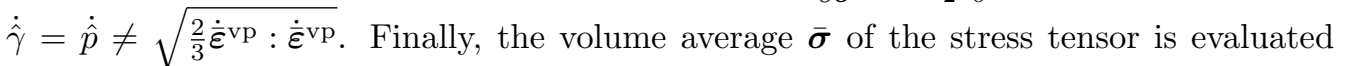
from the accumulated plastic strain $\hat{p}$ following

$$
\overline{\boldsymbol{\sigma}}=\mathbb{C}^{\mathrm{el}}:(\overline{\boldsymbol{\varepsilon}}-\hat{p} \hat{\hat{\boldsymbol{N}}}) .
$$




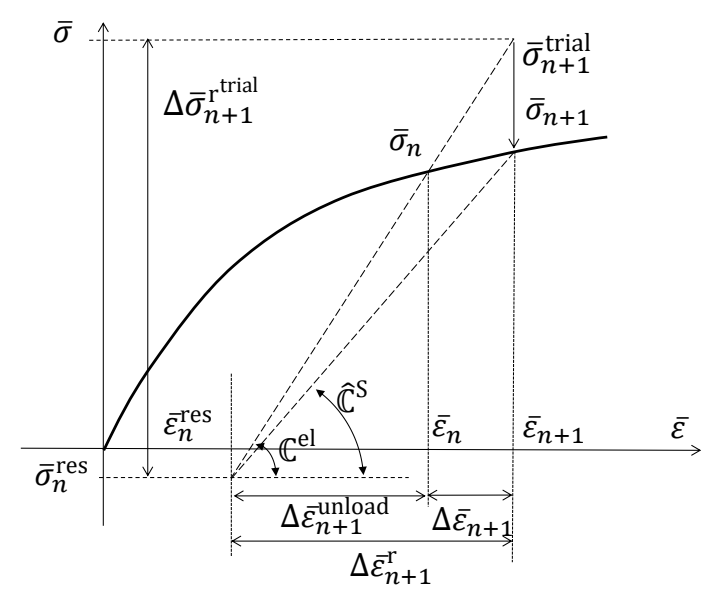

Figure 3: Schematics of the predictor-corrector incremental-secant formulation in each phase.

\subsection{Predictor-corrector equation at volume averaged material level}

Following the idea of incremental-secant process described in Section 2.2, at the beginning of the time interval $\left[t_{n}, t_{n+1}\right]$, a virtual elastic unloading is performed on the composite material, which corresponds to unloading the matrix and inclusions phases to their residual states at time $t_{n}$. Therefore, during that time interval $\left[t_{n}, t_{n+1}\right]$ the elastic predictor in each phase $\omega_{i}$ is evaluated from the residual state as

$$
\overline{\boldsymbol{\sigma}}_{n+1}^{\mathrm{trial}}=\mathbb{C}^{\mathrm{el}}:\left(\overline{\boldsymbol{\varepsilon}}_{n+1}-\overline{\boldsymbol{\varepsilon}}_{n}^{\mathrm{vp}}\right)=\overline{\boldsymbol{\sigma}}_{n}+\mathbb{C}^{\mathrm{el}}: \Delta \overline{\boldsymbol{\varepsilon}}_{n+1}=\overline{\boldsymbol{\sigma}}_{n}^{\mathrm{res}}+\mathbb{C}^{\mathrm{el}}: \Delta \overline{\boldsymbol{\varepsilon}}_{n+1}^{\mathrm{r}} .
$$

Following Fig. 3, we also define

$$
\Delta \overline{\boldsymbol{\sigma}}_{n+1}^{\mathrm{rtrial}}=\overline{\boldsymbol{\sigma}}_{n+1}^{\mathrm{trial}}-\overline{\boldsymbol{\sigma}}_{n}^{\mathrm{res}}=\mathbb{C}^{\mathrm{el}}: \Delta \bar{\varepsilon}_{n+1}^{\mathrm{r}} .
$$

The yielding function (22) is then assessed as follows:

- If the trial stress satisfies the yielding condition

$$
f_{n+1}^{\text {trial }}=\left(\hat{\hat{\sigma}}^{\mathrm{eq}}\right)_{n+1}^{\text {trial }}-\sigma_{\mathrm{Y}}-R\left(\hat{p}_{n}\right) \leq 0,
$$

the loading occurs in the elastic regime, and the elastic predictor is indeed the solution, which leads to

$$
\overline{\boldsymbol{\sigma}}_{n+1}=\overline{\boldsymbol{\sigma}}_{n+1}^{\mathrm{trial}}, \quad \bar{\varepsilon}_{n+1}^{\mathrm{vp}}=\overline{\boldsymbol{\varepsilon}}_{n}^{\mathrm{vp}} \quad \text { and } \quad \hat{p}_{n+1}=\hat{p}_{n} .
$$

- If $f_{n+1}^{\text {trial }}>0$, the stress states are illustrated in Fig. 3, and since visco-plasticity has developed during the time interval, the trial solution should be corrected to satisfy

$$
\Delta \hat{p}=g_{\mathrm{v}}\left(\hat{\hat{\sigma}}^{\mathrm{eq}}, \hat{p}\right) \Delta t,
$$

where $\Delta t=t_{n+1}-t_{n}$. The visco-plastic stress update is written as

$$
\overline{\boldsymbol{\sigma}}_{n+1}=\overline{\boldsymbol{\sigma}}_{n+1}^{\mathrm{trial}}-\mathbb{C}^{\mathrm{el}}: \Delta \bar{\varepsilon}^{\mathrm{vp}}, \text { with } \Delta \bar{\varepsilon}^{\mathrm{vp}}=\Delta \hat{p} \hat{\hat{\hat{N}}}_{n+1},
$$


where, in the incremental secant formulation, the plastic flow direction $\hat{\hat{\mathbf{N}}}_{n+1}$ is approximated by

$$
\hat{\hat{\mathbf{N}}}_{n+1}=\frac{3}{2} \frac{\mathbb{I}^{\mathrm{dev}}:\left(\overline{\boldsymbol{\sigma}}_{n+1}-\overline{\boldsymbol{\sigma}}_{n}^{\mathrm{res}}\right)}{\widehat{\left.\widehat{\widehat{\boldsymbol{\sigma}}}_{n+1}^{-\overline{\boldsymbol{\sigma}}_{n}^{\mathrm{res}}}\right)^{\mathrm{eq}}}}=\frac{3}{2} \frac{\mathbb{I}^{\mathrm{dev}}: \Delta \overline{\boldsymbol{\sigma}}_{n+1}^{\mathrm{r}}}{\Delta \hat{\hat{\sigma}}_{n+1}^{\mathrm{rq}}},
$$

with $\Delta \overline{\boldsymbol{\sigma}}_{n+1}^{\mathrm{r}}$ defined by Eq. (13) and with following Eq. (21)

$$
\Delta \hat{\hat{\sigma}}_{n+1}^{\mathrm{req}}=\sqrt{\frac{3}{2} \mathbb{I}^{\mathrm{dev}}::\left\langle\Delta \boldsymbol{\sigma}_{n+1}^{\mathrm{r}} \otimes \Delta \boldsymbol{\sigma}_{n+1}^{\mathrm{r}}\right\rangle_{\omega_{i}}} .
$$

When considering Eq. (33), it appears that the plastic correction is directed along $\Delta \overline{\boldsymbol{\sigma}}_{n+1}^{\mathrm{r}}$ and not along $\overline{\boldsymbol{\sigma}}_{n+1}$, which is a first order approximation in $\Delta \overline{\boldsymbol{\varepsilon}}^{\mathrm{vp}}$ as discussed by $\mathrm{Wu}$ et al. (2013a) and $\mathrm{Wu}$ et al. (2015a). However, if we neglect the residual stress in the considered phase $\left(\overline{\boldsymbol{\sigma}}_{n}^{\text {res }}=\mathbf{0}\right)$, Eq. (33) can be rewritten as

$$
\hat{\hat{\mathbf{N}}}_{n+1}=\frac{3}{2} \frac{\overline{\boldsymbol{\sigma}}_{n+1}^{\mathrm{dev}}}{\hat{\hat{\sigma}}_{n+1}^{\mathrm{req}}},
$$

in which case the plastic correction is directed along $\overline{\boldsymbol{\sigma}}_{n+1}$.

The resolution of the system of Eqs. (31-34) follows the return mapping algorithm described in the next paragraph, in which the incremental secant operator $\hat{\mathbb{C}}^{\mathrm{S}}$ is directly obtained as an isotropic tensor because of the approximation (33).

\subsection{Return mapping algorithm}

At the trial state, which corresponds to a purely elastic process, the incrementalsecant formulation allows Eq. (33) to be rewritten as

$$
\hat{\hat{\mathbf{N}}}_{n+1}^{\text {trial }}=\frac{3}{2} \frac{\left(\mathbb{C}^{\mathrm{el}}: \Delta \bar{\varepsilon}_{n+1}^{\mathrm{r}}\right)^{\text {dev }}}{3 \mu^{\mathrm{el}} \sqrt{\frac{2}{3} \mathbb{I}^{\mathrm{dev}}::\left\langle\Delta \varepsilon_{n+1}^{\mathrm{r}} \otimes \Delta \varepsilon_{n+1}^{\mathrm{r}}\right\rangle_{\omega_{i}}}}=\frac{3}{2} \frac{\left(\Delta \overline{\boldsymbol{\sigma}}_{n+1}^{\mathrm{rrrial}}\right)^{\text {dev }}}{\Delta \hat{\hat{\sigma}}_{n+1}^{\mathrm{r}} \text { trial }},
$$

where $\mu^{\mathrm{el}}$ is the elastic shear modulus of phase $\omega_{i}$. During this linear elastic process, $\left\langle\Delta \varepsilon_{n+1}^{\mathrm{r}} \otimes \Delta \varepsilon_{n+1}^{\mathrm{r}}\right\rangle_{\omega_{i}}$ in Eq. (36) is computed by using Eq. (7) and reads

$$
\left\langle\Delta \varepsilon_{n+1}^{\mathrm{r}} \otimes \Delta \varepsilon_{n+1}^{\mathrm{r}}\right\rangle_{\omega_{i}}=\frac{1}{v_{i}}\left\langle\Delta \varepsilon_{n+1}^{\mathrm{r}}\right\rangle_{\omega}: \frac{\partial \hat{\mathbb{C}}^{\mathrm{el}}\left(\mathbb{C}_{0}^{\mathrm{el}}, \mathbb{C}_{\mathrm{I}}^{\mathrm{el}}\right)}{\partial \mathbb{C}_{i}^{\mathrm{el}}}:\left\langle\Delta \varepsilon_{n+1}^{\mathrm{r}}\right\rangle_{\omega},
$$

with the composite's effective elastic tensor

$$
\hat{\mathbb{C}}^{\mathrm{el}}\left(\mathbb{C}_{0}^{\mathrm{el}}, \mathbb{C}_{\mathrm{I}}^{\mathrm{el}}\right)=\left[v_{\mathrm{I}} \mathbb{C}_{\mathrm{I}}^{\mathrm{el}}: \mathbb{B}^{\epsilon}+v_{0} \mathbb{C}_{0}^{\mathrm{el} l}\right]:\left[v_{\mathrm{I}} \mathbb{B}^{\epsilon}+v_{0} \mathbb{I}\right]^{-1} .
$$

Since $\mathbb{C}^{\mathrm{el}}$ is isotropic we have $\mathbb{C}^{\mathrm{el}}:\left(\Delta \hat{p} \hat{\hat{\mathbf{N}}}_{n+1}\right)=2 \mu^{\mathrm{el}} \hat{\hat{\mathbf{N}}}_{n+1} \Delta \hat{p}$, and Eq. (32) is rewritten

$$
\left(\overline{\boldsymbol{\sigma}}_{n+1}-\overline{\boldsymbol{\sigma}}_{n}^{\mathrm{res}}\right)^{\mathrm{dev}}=\left(\overline{\boldsymbol{\sigma}}_{n+1}^{\mathrm{trial}}-\overline{\boldsymbol{\sigma}}_{n}^{\mathrm{res}}\right)^{\mathrm{dev}}-2 \mu^{\mathrm{el}} \hat{\hat{\mathbf{N}}}_{n+1} \Delta \hat{p} .
$$


Using the definitions (36) and (39), one has

$$
\left[\Delta \hat{\hat{\sigma}}_{n+1}^{\mathrm{req}}+3 \mu^{\mathrm{el}} \Delta \hat{p}\right] \hat{\hat{\mathbf{N}}}_{n+1}=\Delta \hat{\hat{\sigma}}_{n+1}^{\mathrm{r}}{ }_{n+1}^{\text {trial }}{ }^{\mathrm{eq}} \hat{\hat{N}}_{n+1}^{\text {trial }} .
$$

Enforcing that both normal tensors in Eq. (40) have the same norm, i.e. $\hat{\hat{\mathbf{N}}}_{n+1}: \hat{\hat{\mathbf{N}}}_{n+1}=$ $\hat{\hat{\mathbf{N}}}_{n+1}^{\text {trial }}: \hat{\hat{\mathbf{N}}}_{n+1}^{\text {trial }}$, this relation results in

$$
\begin{aligned}
& \Delta \hat{\hat{\sigma}}_{n+1}^{\mathrm{req}}+3 \mu^{\mathrm{el}} \Delta \hat{p}=\Delta \hat{\hat{\sigma}}_{n+1}^{\mathrm{r}}{ }_{n+1}^{\mathrm{rial}} \text {, and } \\
& \hat{\hat{\mathbf{N}}}_{n+1}=\hat{\hat{\mathbf{N}}}_{n+1}^{\text {trial }} \text {. }
\end{aligned}
$$

Since in the incremental-secant process the trial state is computed from a virtual unloaded/residual state, we adopt a last assumption which is

$$
\hat{\hat{\sigma}}_{n+1}^{\mathrm{eq}} \simeq \Delta \hat{\hat{\sigma}}_{n+1}^{\mathrm{r}},
$$

which gives good predictions especially when the norm of the residual stress, which follows the unloading $\overline{\boldsymbol{\sigma}}_{n}^{\text {res }}$ is much smaller in comparison to the norm of the previous stress $\overline{\boldsymbol{\sigma}}_{n}$, see Fig. 2. ${ }^{1}$ However, this assumption has an obvious drawback. From some material systems, after using a virtual elastic unloading, the residual stress in the elasto-viscoplastic phases can remain a high level, i.e. the norm of the residual tensor is important. In this case, the assumption (43) is not accurate anymore and leads to poor predictions.

For the volume averaged material, the visco-plastic correction consists in solving the system of Eqs. (31) and (41), which can be restated as

$$
\begin{aligned}
& F_{p}=\Delta \hat{p}-g_{\mathrm{v}}\left(\hat{\hat{\sigma}}_{n+1}^{\mathrm{eq}}, \hat{p}_{n+1}\right) \Delta t=0, \\
& F_{\sigma}=\hat{\hat{\sigma}}_{n+1}^{\mathrm{eq}}+3 \mu^{\mathrm{el}} \Delta \hat{p}-\Delta \hat{\hat{\sigma}}_{n+1}^{\mathrm{r}}{ }_{n+1}^{\mathrm{eq}}=0 .
\end{aligned}
$$

These two equations can be solved iteratively with a Newton-Raphson method, which is expressed at iteration as

$$
\begin{aligned}
0-F_{p} & =\frac{\partial F_{p}}{\partial \hat{p}} \delta \hat{p}+\frac{\partial F_{p}}{\partial \hat{\hat{\sigma}}^{\mathrm{eq}}} \delta \hat{\hat{\sigma}}^{\mathrm{eq}} \\
0-F_{\sigma} & =\frac{\partial F_{\sigma}}{\partial \hat{p}} \delta \hat{p}+\frac{\partial F_{\sigma}}{\partial \hat{\hat{\sigma}}^{\mathrm{eq}}} \delta \hat{\hat{\sigma}}^{\mathrm{eq}}
\end{aligned}
$$

or, using Eq. (45), as

$$
\begin{aligned}
& 0=F_{p}+\left(1-\frac{\partial g_{\mathrm{v}}}{\partial \hat{p}} \Delta t\right) \delta \hat{p}-\frac{\partial g_{\mathrm{v}}}{\partial \hat{\hat{\sigma}}^{\mathrm{eq}}} \Delta t \delta \hat{\hat{\sigma}}^{\mathrm{eq}} \\
& 0=F_{\sigma}+3 \mu^{\mathrm{el}} \delta \hat{p}+\delta \hat{\sigma}^{\mathrm{eq}} .
\end{aligned}
$$

\footnotetext{
${ }^{1}$ Another approximation was proposed in (Wu et al., 2015a) for elasto-plasticity, i.e.

$$
\begin{aligned}
& \left(\hat{\hat{\sigma}}_{n+1}^{\text {trial }}{ }^{\text {eq }}\right)^{2} \simeq\left(\hat{\hat{\sigma}}_{n}^{\text {eq }}\right)^{2}-\left(\Delta \hat{\hat{\sigma}}_{n+1}^{\text {unload }}{ }^{\text {eq }}\right)^{2}-3 \overline{\boldsymbol{\sigma}}_{n}: \mathbb{I}^{\text {dev }}: \Delta \overline{\boldsymbol{\sigma}}_{n}^{\text {unload }}+ \\
& \left(\Delta \hat{\hat{\sigma}}_{n+1}^{\mathrm{rtrial}}{ }^{\mathrm{eq}}\right)^{2}+3 \overline{\boldsymbol{\sigma}}_{n}^{\mathrm{res}}: \mathbb{I}^{\mathrm{dev}}: \Delta \overline{\boldsymbol{\sigma}}_{n+1}^{\mathrm{rtrial}},
\end{aligned}
$$

where $\Delta \overline{\boldsymbol{\sigma}}_{n+1}^{\text {rtrial }}$ has been defined in Eq. 28. For complex loading history, the simple approximation (43) shows better consistency. 
Substituting the expression of $\delta \hat{\hat{\sigma}}^{\mathrm{eq}}$ from equation (49) into equation (48) leads to

$$
\delta \hat{p}=-\frac{1}{h_{\mathrm{v}}}\left(\frac{F_{p}}{\left(\partial g_{\mathrm{v}} / \partial \hat{\hat{\sigma}}^{\mathrm{eq}}\right) \Delta t}+F_{\sigma}\right)
$$

with

$$
h_{\mathrm{v}}=\frac{1}{\left(\partial g_{\mathrm{v}} / \partial \hat{\hat{\sigma}}^{\mathrm{eq}}\right) \Delta t}-\frac{\left(\partial g_{\mathrm{v}} / \partial \hat{p}\right)}{\left(\partial g_{\mathrm{v}} / \partial \hat{\hat{\sigma}}^{\mathrm{eq}}\right)}+3 \mu^{\mathrm{el}} .
$$

We need to emphasize the following two points:

- According to the Mori-Tanaka scheme, the stress and strain in the inclusions phase $\omega_{\text {I }}$ remain uniform. Therefore, the second statistical moment estimate of the von Mises stress in the inclusions is equal to its first statistical moment estimate and reads

$$
\hat{\hat{\sigma}}_{\mathrm{I}}^{\mathrm{eq}}=\bar{\sigma}_{\mathrm{I}}^{\mathrm{eq}}=\sqrt{\frac{3}{2} \overline{\boldsymbol{\sigma}}_{\mathrm{I}}^{\mathrm{dev}}: \overline{\boldsymbol{\sigma}}_{\mathrm{I}}^{\mathrm{dev}}} .
$$

Assumption (43) is thus not required in the inclusions phases. For computational efficiency we use the incremental-secant method with first statistical moment estimates in the inclusions phase since it yields the same predictions.

- In the matrix phase $\omega_{0}$, if we replace the second statistical moment estimate of the von Mises stress, $\hat{\hat{\sigma}}_{0}^{\text {eq }}$, everywhere in this section by its first statistical moment estimate, $\bar{\sigma}_{0}^{\mathrm{eq}}$, we straightforwardly retrieve the incremental-secant formulation of the volume averaged material based on the first statistical moment estimate.

\subsection{Incremental-secant operator}

The secant operators $\hat{\mathbb{C}}^{\mathrm{S}}$ of the averaged phase material, defined in Eq. (11), can now be evaluated.

In the case of the absence of visco-plastic flow, i.e. if $f_{n+1}^{\text {trial }} \leq 0$, the incremental secant operator is directly obtained as $\hat{\mathbb{C}}^{\mathrm{S}}=\mathbb{C}^{\mathrm{el}}$.

In the case of plastic flow, i.e. if $f_{n+1}^{\text {trial }}>0$, according to the approximation of $\hat{\hat{\mathbf{N}}}_{n+1}$, Eq. (33), the incremental secant operator $\hat{\mathbb{C}}_{n+1}^{\mathrm{S}}$ satisfies,

$$
\Delta \overline{\boldsymbol{\sigma}}_{n+1}^{\mathrm{r}}=\hat{\mathbb{C}}_{n+1}^{\mathrm{S}}: \Delta \bar{\varepsilon}_{n+1}^{\mathrm{r}}=\mathbb{C}^{\mathrm{el}}: \Delta \bar{\varepsilon}_{n+1}^{\mathrm{r}}-2 \mu^{\mathrm{el}} \Delta \hat{p} \hat{\hat{N}}_{n+1}
$$

which becomes after using Eqs. (36) and (42),

$$
\hat{\mathbb{C}}_{n+1}^{\mathrm{S}}: \Delta \bar{\varepsilon}_{n+1}^{\mathrm{r}}=\left[\mathbb{C}^{\mathrm{el}}-3 \mu^{\mathrm{el}} \Delta \hat{p} \frac{\mathbb{I}^{\mathrm{dev}}: \mathbb{C}^{\mathrm{el}}}{\Delta \hat{\hat{\sigma}}_{n+1}^{\mathrm{r}}{ }_{n+1}^{\mathrm{eq}}}\right]: \Delta \bar{\varepsilon}_{n+1}^{\mathrm{r}} .
$$

For $\mathrm{J}_{2}$-EVP, since $\mathbb{C}^{\mathrm{el}}$ is isotropic, the incremental-secant operator $\hat{\mathbb{C}}^{\mathrm{S}}$ is also isotropic. Moreover, as $\mathbb{C}^{\mathrm{el}}=3 \kappa^{\mathrm{el}} \mathbb{I}^{\mathrm{vol}}+2 \mu^{\mathrm{el}} \mathbb{I}^{\mathrm{dev}}$ for phase $\omega_{i}$, this operator can be evaluated as

$$
\hat{\mathbb{C}}^{\mathrm{S}}=3 \kappa^{\mathrm{el}} \mathbb{I}^{\mathrm{vol}}+2 \hat{\mu}^{\mathrm{S}} \mathbb{I}^{\mathrm{dev}},
$$


with

$$
\hat{\mu}^{\mathrm{S}}=\mu^{\mathrm{el}}-\frac{3 \mu^{\mathrm{el}^{2}} \Delta \hat{p}}{\Delta \hat{\hat{\sigma}}^{\mathrm{r}}{ }_{n+1}^{\mathrm{trial}}},
$$

where $\kappa^{\mathrm{el}}$ and $\mu^{\mathrm{el}}$ are the elastic bulk and shear moduli of phase $\omega_{i}$, respectively. From Eq. (55) we can see that the incremental secant operator reduces to the elastic tensor when the phase is loaded elastically $(\Delta \hat{p}=0)$.

The incremental-secant operator $\hat{\mathbb{C}}^{\mathrm{S}}$ of the phase thus depends on the volume average on the phase of the strain increments $\Delta \bar{\varepsilon}^{\mathrm{r}}$ but also on the volume average on the composite material strain increment $\left\langle\Delta \varepsilon_{n+1}^{\mathrm{r}}\right\rangle_{\omega}$, because of the evaluation of Eq. (37). Two algorithmic operators are thus required: $\frac{\partial \hat{\mathbb{C}}_{n+1}^{S}}{\partial \bar{\varepsilon}_{n+1}^{\mathrm{r}}}$ and $\frac{\partial \hat{\mathbb{C}}_{n+1}^{\mathrm{S}}}{\partial\left\langle\Delta \varepsilon_{n+1}^{\mathrm{r}}\right\rangle_{\omega}}$, which were evaluated by Wu et al. (2015a) and are reported in Appendix A.1.

\section{Mean-Field Homogenization based on the incremental-secant approach}

In the previous Section, we have described the resolution of the $\mathrm{J}_{2}$-EVP constitutive equations, which were volume averaged on the phase $\omega_{i}$ of the composite material. The resolution of these equations, for each phase $\omega_{i}$, is thus implemented as a constitutive box, in which the stress $\overline{\boldsymbol{\sigma}}_{i n+1}^{\mathrm{r}}$ and secant operator $\widehat{\mathbb{C}}_{i n+1}^{\mathrm{S}}$ are evaluated from $\Delta \bar{\varepsilon}_{i n+1}^{\mathrm{r}}$, from the residual stress-strain state $\left(\overline{\boldsymbol{\sigma}}_{i n}^{\text {res }}, \overline{\boldsymbol{\varepsilon}}_{i n}^{\text {res }}\right)$ reached upon the composite material virtual unloading, from the phase internal variables $\eta_{i}$, such as $\hat{p}$, but also from the composite material strain increment $\Delta \bar{\varepsilon}_{n+1}^{\mathrm{r}}$ in order to evaluate Eq. (37).

The set of Eqs. (11-13) is thus summarized by

$$
\overline{\boldsymbol{\sigma}}_{i n+1}=\mathcal{F}_{i}\left(\Delta \bar{\varepsilon}_{i n+1}^{\mathrm{r}}, \Delta \bar{\varepsilon}_{n+1}^{\mathrm{r}} ; \eta_{i n}, \bar{\varepsilon}_{i n}^{\mathrm{res}}, \overline{\boldsymbol{\sigma}}_{i n}^{\mathrm{res}}\right),
$$

and

$$
\hat{\mathbb{C}}_{i n+1}^{\mathrm{S}}=\mathcal{G}_{i}\left(\Delta \bar{\varepsilon}_{i n+1}^{\mathrm{r}}, \Delta \bar{\varepsilon}_{n+1}^{\mathrm{r}} ; \eta_{i n}, \bar{\varepsilon}_{i n}^{\mathrm{res}}, \overline{\boldsymbol{\sigma}}_{i n}^{\mathrm{res}}\right) .
$$

Finally, two algorithmic operators are also obtained from the constitutive box:

$$
\begin{aligned}
\hat{\mathbb{C}}_{i n+1}^{\mathrm{algo}} & =\frac{\partial \overline{\boldsymbol{\sigma}}_{i n+1}}{\partial \overline{\boldsymbol{\varepsilon}}_{i n+1}^{\mathrm{r}}}=\hat{\mathbb{C}}_{i n+1}^{\mathrm{S}}+\Delta \bar{\varepsilon}_{i n+1}^{\mathrm{r}}: \frac{\partial \hat{\mathbb{C}}_{i n+1}^{\mathrm{S}}}{\partial \bar{\varepsilon}_{i n+1}^{\mathrm{r}}}, \text { and } \\
\hat{\mathbb{C}}_{i c n+1}^{\mathrm{algo}} & =\frac{\partial \overline{\boldsymbol{\sigma}}_{i n+1}}{\partial \overline{\boldsymbol{\varepsilon}}_{n+1}^{\mathrm{r}}}=\Delta \bar{\varepsilon}_{i n+1}^{\mathrm{r}}: \frac{\partial \hat{\mathbb{C}}_{i n+1}^{\mathrm{S}}}{\partial \bar{\varepsilon}_{n+1}^{\mathrm{r}}} .
\end{aligned}
$$

The incremental-secant MFH process now strictly follows the method developed for EP materials by (Wu et al., 2013a, 2015a) and is summarized as follows for the time interval $\left[t_{n} t_{n+1}\right]$ :

1. A virtual unloading is applied at the composite material level so that $\overline{\boldsymbol{\sigma}}_{n}^{\text {res }}=0$, using the composite material elastic tensor (38). The residual strain-stress states are then evaluated as

- At the composite material level, beside $\overline{\boldsymbol{\sigma}}_{n}^{\text {res }}=0$, the residual strain reads

$$
\bar{\varepsilon}_{n}^{\mathrm{res}}=\bar{\varepsilon}_{n}-\Delta \bar{\varepsilon}_{n}^{\text {unload }}=\bar{\varepsilon}_{n}-\hat{\mathbb{C}}^{\mathrm{el}-1}: \overline{\boldsymbol{\sigma}}_{n+1} .
$$


- Using the virtual unloading increment obtained in Eq. (60), the residual state in the phase $\omega_{i}$ is obtained by using the MFH equations (1-3), yielding for the inclusions phase $\omega_{\text {I }}$

$$
\begin{aligned}
& \bar{\varepsilon}_{\mathrm{I} n}^{\text {res }}=\bar{\varepsilon}_{\mathrm{I} n}-\mathbb{B}^{\epsilon}:\left[v_{\mathrm{I}} \mathbb{B}^{\epsilon}+v_{0} \mathbb{I}\right]^{-1}: \Delta \bar{\varepsilon}_{n}^{\text {unload }}, \\
& \overline{\boldsymbol{\sigma}}_{\mathrm{I} n}^{\text {res }}=\overline{\boldsymbol{\sigma}}_{\mathrm{I} n}-\mathbb{C}_{\mathrm{I}}^{\mathrm{el}}: \mathbb{B}^{\epsilon}:\left[v_{\mathrm{I}} \mathbb{B}^{\epsilon}+v_{0} \mathbb{I}\right]^{-1}: \Delta \bar{\varepsilon}_{n}^{\text {unload }},
\end{aligned}
$$

and for the matrix phase $\omega_{0}$

$$
\begin{aligned}
& \bar{\varepsilon}_{0 n}^{\text {res }}=\bar{\varepsilon}_{0 n}-\left[v_{\mathrm{I}} \mathbb{B}^{\epsilon}+v_{0} \mathbb{I}\right]^{-1}: \Delta \bar{\varepsilon}_{n}^{\text {unload }}, \\
& \overline{\boldsymbol{\sigma}}_{0 n}^{\text {res }}=\overline{\boldsymbol{\sigma}}_{0 n}-\mathbb{C}_{0}^{\mathrm{el}}:\left[v_{\mathrm{I}} \mathbb{B}^{\epsilon}+v_{0} \mathbb{I}\right]^{-1}: \Delta \overline{\boldsymbol{\varepsilon}}_{n}^{\text {unload }} .
\end{aligned}
$$

2. The composite material secant strain increment is thus computed from Eq. (10), with

$$
\Delta \bar{\varepsilon}_{n+1}^{\mathrm{r}}=\bar{\varepsilon}_{n}+\Delta \bar{\varepsilon}_{n+1}-\bar{\varepsilon}_{n}^{\mathrm{res}} .
$$

3. The incremental-secant MFH equations (11-13) are rewritten

$$
\begin{aligned}
\Delta \bar{\varepsilon}_{n+1}^{\mathrm{r}} & =v_{0} \Delta \bar{\varepsilon}_{0 n+1}^{\mathrm{r}}+v_{\mathrm{I}} \Delta \bar{\varepsilon}_{\mathrm{I}_{n+1}^{\mathrm{r}}}^{\mathrm{r}}, \\
\overline{\boldsymbol{\sigma}}_{n+1} & =v_{0} \overline{\boldsymbol{\sigma}}_{0 n+1}+v_{\mathrm{I}} \overline{\boldsymbol{\sigma}}_{\mathrm{I} n+1}=v_{0} \Delta \overline{\boldsymbol{\sigma}}_{0 n+1}^{\mathrm{r}}+v_{\mathrm{I}} \Delta \overline{\boldsymbol{\sigma}}_{\mathrm{I} n+1}^{\mathrm{r}}, \\
\Delta \bar{\varepsilon}_{\mathrm{I}}^{\mathrm{r}}{ }_{n+1} & =\mathbb{B}^{\epsilon}\left(\mathrm{I}, \hat{\mathbb{C}}_{0}^{\mathrm{S}}, \hat{\mathbb{C}}_{\mathrm{I}}^{\mathrm{S}}\right): \Delta \bar{\varepsilon}_{0 n+1}^{\mathrm{r}} .
\end{aligned}
$$

and are solved iteratively by calling the constitutive box (57-58). The M-T iterative process has been detailed by Wu et al. (2015a) and is summarized as follows

- The strain increment in the inclusions phase is initialized by $\Delta \bar{\varepsilon}_{n+1}^{\mathrm{r}} \rightarrow \Delta \bar{\varepsilon}_{\mathrm{I} n+1}^{\mathrm{r}}$.

- The constitutive box, Eqs. (57-58), is called by each phase $\omega_{i}$.

- A residual function was built from Eq. (62) by Wu et al. (2013a) as

$$
\begin{aligned}
\boldsymbol{F}= & \hat{\mathbb{C}}_{0 n+1}^{\mathrm{S}}:\left[\Delta \bar{\varepsilon}_{\mathrm{I} n+1}^{\mathrm{r}}-\frac{1}{v_{0}} \mathbb{S}^{-1}:\left(\Delta \bar{\varepsilon}_{\mathrm{I} n+1}^{\mathrm{r}}-\Delta \bar{\varepsilon}_{n+1}^{\mathrm{r}}\right)\right]- \\
& \hat{\mathbb{C}}_{\mathrm{I} n+1}^{\mathrm{S}}: \Delta \bar{\varepsilon}_{\mathrm{I} n+1}^{\mathrm{r}},
\end{aligned}
$$

with the Jacobian computed at constant $\Delta \bar{\varepsilon}_{n+1}^{\mathrm{r}}$

$$
\mathbb{J}=\frac{\partial \boldsymbol{F}}{\partial \bar{\varepsilon}_{\mathrm{I}}}+\frac{\partial \boldsymbol{F}}{\partial \bar{\varepsilon}_{0}}:\left.\frac{\partial \bar{\varepsilon}_{0}}{\partial \bar{\varepsilon}_{\mathrm{I}}}\right|_{\Delta \bar{\varepsilon}_{n+1}^{\mathrm{r}}}=\frac{\partial \boldsymbol{F}}{\partial \bar{\varepsilon}_{\mathrm{I}}}-\frac{v_{\mathrm{I}}}{v_{0}} \frac{\partial \boldsymbol{F}}{\partial \bar{\varepsilon}_{0}},
$$

detailed in Appendix A.2. The volume averaged strain increment in the inclusions phase $\omega_{i}$ is then updated as

$$
\Delta \bar{\varepsilon}_{\mathrm{I} n+1}^{\mathrm{r}} \leftarrow \Delta \bar{\varepsilon}_{\mathrm{I} n+1}^{\mathrm{r}}-\mathbb{J}^{-1}: \boldsymbol{F},
$$

until convergence is reached. 
4. After convergence, the composite material algorithmic operator is constructed from Eq. (67), yielding

$$
\hat{\mathbb{C}}_{n+1}^{\text {algo }}=v_{\mathrm{I}}\left(\hat{\mathbb{C}}_{\mathrm{I} n+1}^{\text {algo }}: \frac{\partial \bar{\varepsilon}_{\mathrm{I}}}{\partial \bar{\varepsilon}}+\hat{\mathbb{C}}_{\mathrm{Ic} n+1}^{\text {algo }}\right)+v_{0}\left(\hat{\mathbb{C}}_{0 n+1}^{\text {algo }}: \frac{\partial \bar{\varepsilon}_{0}}{\partial \bar{\varepsilon}}+\hat{\mathbb{C}}_{0 \mathrm{c} n+1}^{\text {algo }}\right) .
$$

The phase consistent material operators were defined in Eq. (59), and the last two terms $\frac{\partial \bar{\varepsilon}_{\mathrm{I}}}{\partial \bar{\varepsilon}}$ and $\frac{\partial \bar{\varepsilon}_{\mathrm{I}}}{\partial \bar{\varepsilon}}$ are obtained by recalling that since $\boldsymbol{F}=0$, the variation of the strain increment in each phase due to a variation $\delta \Delta \bar{\varepsilon}$ is obtained by constraining $\delta \boldsymbol{F}=\frac{\partial \boldsymbol{F}}{\partial \bar{\varepsilon}_{\mathrm{I}}}: \delta \Delta \bar{\varepsilon}_{\mathrm{I}}^{\mathrm{r}}+\frac{\partial \boldsymbol{F}}{\partial \overline{\boldsymbol{\varepsilon}}_{0}}: \delta \Delta \bar{\varepsilon}_{0}^{\mathrm{r}}+\frac{\partial \boldsymbol{F}}{\partial \overline{\boldsymbol{\varepsilon}}}: \delta \Delta \bar{\varepsilon}^{\mathrm{r}}=0$. Using Eq. (66), one thus has

$$
\begin{aligned}
\frac{\partial \bar{\varepsilon}_{\mathrm{I}}}{\partial \bar{\varepsilon}} & =-\mathbb{J}^{-1}: \frac{\partial \boldsymbol{F}}{\partial \bar{\varepsilon}}, \text { and } \\
\frac{\partial \bar{\varepsilon}_{0}}{\partial \bar{\varepsilon}} & =\frac{1}{v_{0}}\left(\mathbb{I}-v_{\mathrm{I}} \frac{\partial \bar{\varepsilon}_{\mathrm{I}}}{\partial \bar{\varepsilon}}\right) .
\end{aligned}
$$

The expression of the missing term $\frac{\partial \boldsymbol{F}}{\partial \bar{\varepsilon}}$ is detailed in Appendix A.2.

Clearly, the particularities related to the EVP behaviors are inherent to the constitutive box (57-58), while the incremental-secant MFH resolution remains independent of the visco-plastic behavior.

\section{Applications}

The predictions accuracy of the incremental-secant MFH process is assessed in this section. In particular we consider the formulation using the second statistical moment estimate of the von Mises stress based on Eq. (43). These predictions are compared to a version in which only the first statistical moment estimate is considered. This is readily achieved by replacing in both phases the second statistical moment estimate of the von Mises stress, $\hat{\hat{\sigma}}_{i}^{\text {eq }}$, everywhere in the formalism by its first statistical moment estimate, $\bar{\sigma}_{i}^{\text {eq }}$. However, for that latter case to remain accurate in the case of hard inclusions, the residual stress in the matrix phase $\overline{\boldsymbol{\sigma}}_{0 n}^{\text {res }}$ has to be canceled after the composite virtual unloading. In the inclusions phase, the residual stress is always considered. This point has been discussed by $\mathrm{Wu}$ et al. (2013a) for EP composite materials. When the residual stress in the matrix phase is canceled we denote the secant operator by $C_{0}^{0}$, and if this not the case, i.e. if the residual stress in the matrix is kept, the secant operator is denoted by $C_{0}^{\mathrm{r}}$.

Several material systems such as spherical inclusions or short fibers embedded in a matrix are considered. The tests involved different triaxiality conditions, with monotonic and non-radial loading conditions. The results are compared to finite element (FE) results, to Fast-Fourier-Transform (FFT) results, to experiments, and to other homogenization predictions.

\subsection{Elastic short fiber-reinforced elasto-plastic matrix}




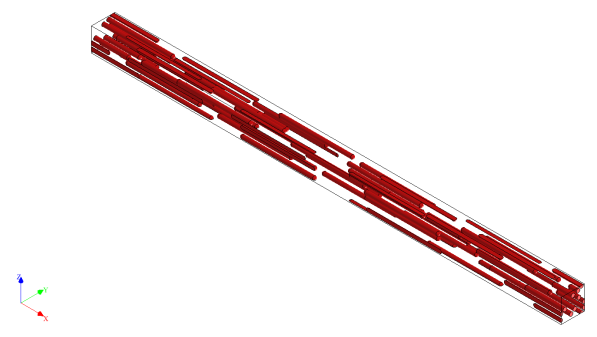

(a) RVE for $0^{\circ}$ and $90^{\circ}$-uniaxial loading

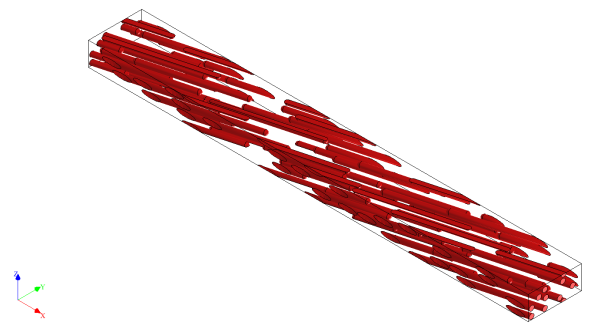

(c) RVE for $15^{\circ}$-uniaxial loading

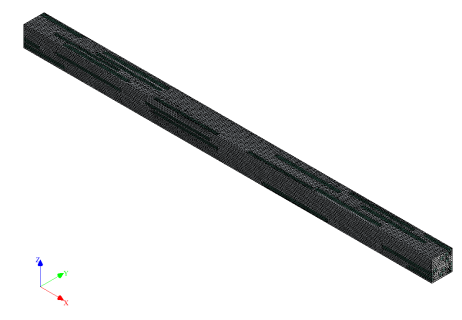

(b) Mesh for $0^{\circ}$ and $90^{\circ}$-uniaxial loading

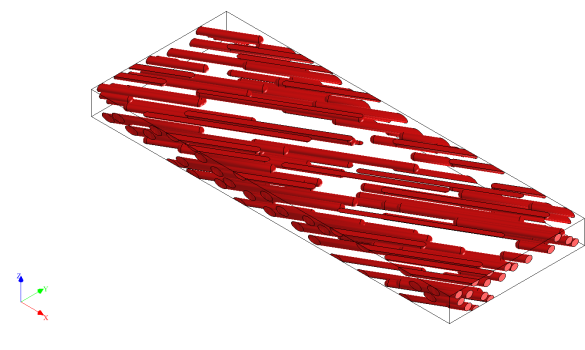

(d) RVE for $30^{\circ}$-uniaxial loading

Figure 4: RVEs for the short fiber-reinforced EP matrix under different loading conditions. (a) RVE geometry for uniaxial loading along the short fiber direction $\left(0^{\circ}\right)$ and transverse $\left(90^{\circ}\right)$ to the short fiber direction, and (b) corresponding FE mesh. (c) RVE geometry fo uniaxial loading directed at $15^{\circ}$ to the short fiber direction. (d) RVE geometry for uniaxial loading directed at $30^{\circ}$ to the short fiber direction.

First, the method accuracy is discussed in the case of an elasto-plastic matrix reinforced by short fibers. The matrix is modeled using a $\mathrm{J}_{2}$-EP model with the linearexponential hardening law described by

$$
R=h_{1} p+h_{2}(1-\exp (-n p)),
$$

where $h_{1}$ and $h_{2}$ are the hardening coefficients, and where $n$ is the hardening exponent. The material properties of the two phases read:

- Matrix: Elastic Young's modulus $E_{0}^{\mathrm{el}}=3 \mathrm{GPa}$; Poisson ratio $\nu_{0}=0.35$; yield stress $\sigma_{\mathrm{Y}_{0}}=20 \mathrm{MPa}$; first hardening coefficient $h_{1_{0}}=1 \mathrm{MPa}$; second hardening coefficient $h_{2_{0}}=15 \mathrm{MPa}$; hardening exponent $n_{0}=150$.

- Inclusions: Elastic Young's modulus $E_{\mathrm{I}}^{\mathrm{el}}=72 \mathrm{GPa}$; Poisson ratio $\nu_{\mathrm{I}}=0.2$; volume fraction $v_{\mathrm{I}}=15.87 \%$; and aspect ratio of $\alpha_{\mathrm{I}}=20$.

Different monotonic loading conditions corresponding to different triaxiality states are first considered:

Loading \#1: Uniaxial loading in a direction oriented at $0^{\circ}, 15^{\circ}, 30^{\circ}$, and $90^{\circ}$ to the short fiber direction;

Loading \#2: In-plane biaxial loading $\bar{\varepsilon}_{11}=\bar{\varepsilon}_{22}$ with $\bar{\sigma}_{33}=0$ and with the direction 1 along the fiber direction. 


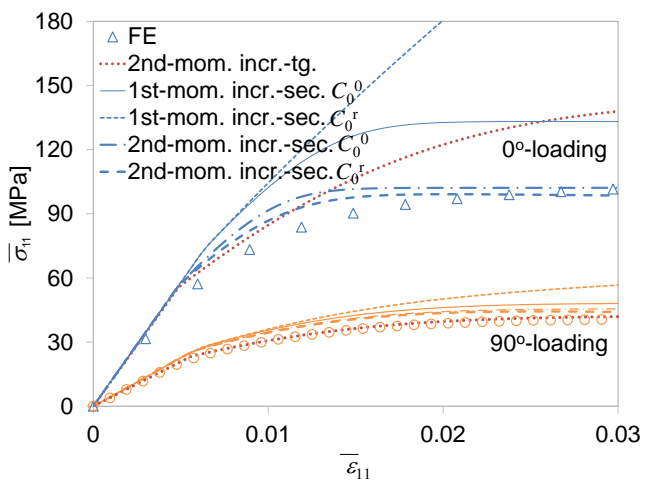

(a) $0^{\circ}$ and $90^{\circ}$-uniaxial loading conditions

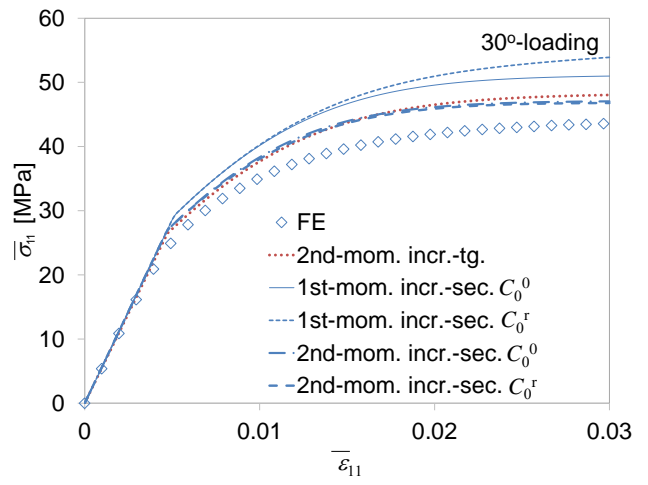

(c) $30^{\circ}$-uniaxial loading condition

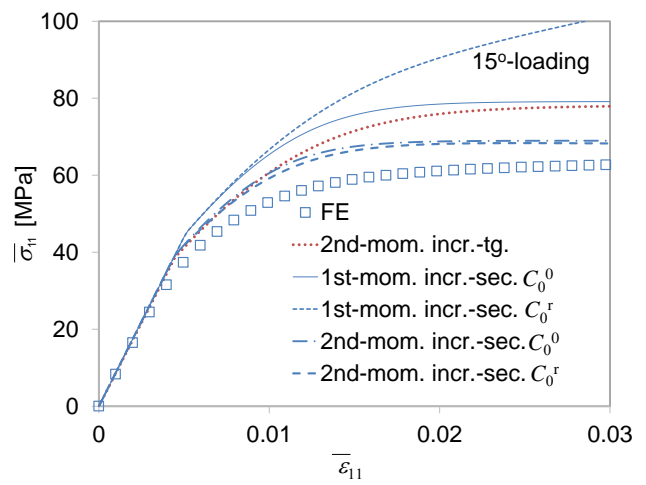

(b) $15^{\circ}$-uniaxial loading condition

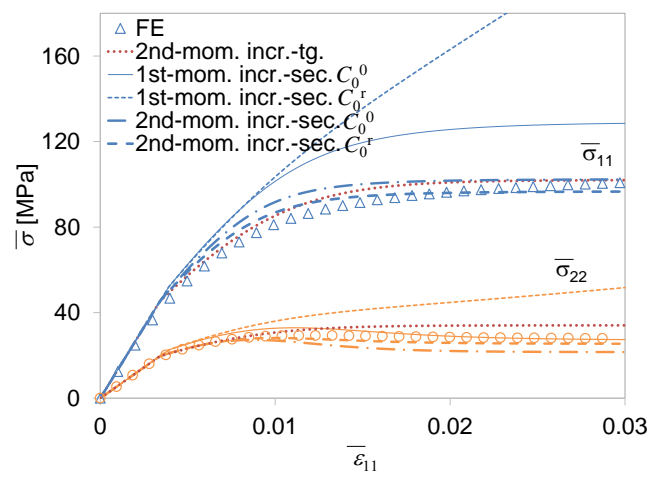

(d) Biaxial loading condition

Figure 5: Short fiber-reinforced EP matrix under different loading conditions The method predictions are compared with FE results and the incremental tangent method. (a) Uniaxial loading along the short fiber direction $\left(0^{\circ}\right)$ and transverse $\left(90^{\circ}\right)$ to the short fiber direction. (b) Uniaxial loading directed at $15^{\circ}$ to the short fiber direction. (c) Uniaxial loading directed at $30^{\circ}$ to the short fiber direction. (d) Biaxial loading. Direction 1 is along the fiber direction.

The reference results are obtained using the FE method on the RVE geometries illustrated in Fig. 4. Each RVE includes 50 complete fibers, which are divided when they intersect a RVE face, yielding 79, 105, and 115 inclusions bodies for respectively the RVE with the fibers oriented at $0^{\circ}$ and $90^{\circ}$ with respect to the loading direction, see Fig. 4(a), the RVE with the fibers oriented at $15^{\circ}$ with respect to the loading direction, see Fig. 4(c), and the RVE with the fibers oriented at $30^{\circ}$ with respect to the loading direction, see Fig. 4(d). For illustration purpose, a 411,180-node FE mesh is illustrated in Fig. 4(b) for the RVE with the fibers oriented at $0^{\circ}$ and $90^{\circ}$ with respect to the loading direction.

Figure 5 compares the predictions obtained using the incremental-secant approach with first statistical moment estimates and with second statistical moment estimates, to the results obtained with the incremental-tangent approach with second statistical moment estimates, and to full-field finite element (FE) predictions. When considering the incremental-secant $\mathrm{MFH}$, in both cases of first statistical moment estimates and of 
second statistical moment estimates, we successively consider the residual stress in the matrix phase $\left(C_{0}^{\mathrm{r}}\right.$ secant operator) and neglect it $\left(C_{0}^{0}\right.$ secant operator $)$. On the one hand, when considering the first statistical moment estimates, keeping the residual stress in the matrix phase leads to unacceptable solutions. On the other hand, when considering the second statistical moment estimates, the solutions obtained with or without considering the residual stress in the matrix phase are of comparable accuracy. It can also be seen in Fig. 5 that when comparing the different homogenization methods with second statistical moment estimates that the incremental-secant method improves the predictions as compared to the incremental-tangent approach when considering uniaxial loading conditions, see Figs. 5(a)-5(c), in particular when the loading direction is close to the fiber direction. Moreover, when considering the biaxial loading condition, see Fig. 5(d), the incremental-secant approach captures the decrease of the stress in the transverse direction, accordingly to the finite element predictions.

\subsection{Elastic short fiber-reinforced elasto-visco-plastic matrix}

\subsubsection{Polymer with a Perzyna's visco-plastic model reinforced with short glass fibers}

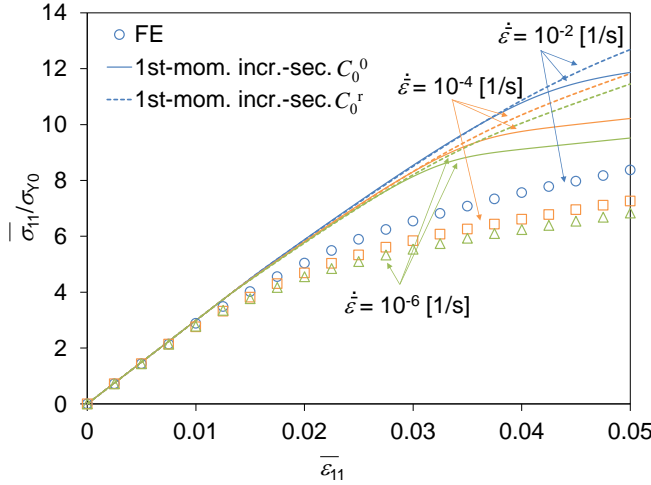

(a) 1st moments

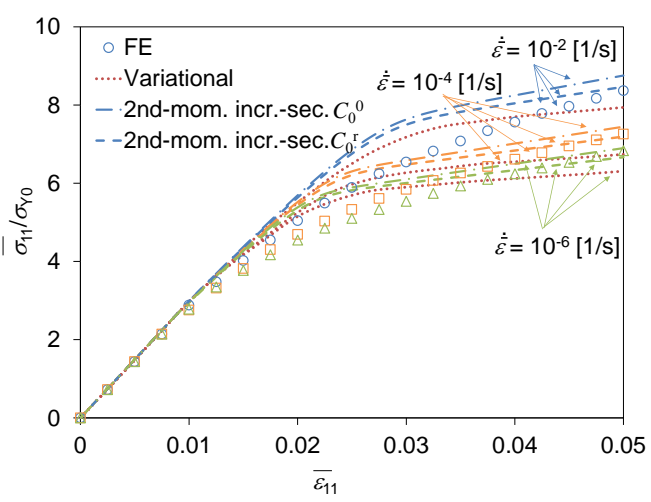

(b) 2nd moments

Figure 6: Short glass fiber-reinforced polyamide under uniaxial loading with a matrix material following the Perzyna's visco-plastic model. The method predictions for different strain rates using (a) first statistical moment estimates and (b) second statistical moment estimates are compared with the FE results and with the prediction of the variational updates formulation provided by Brassart et al. (2012).

The aligned short glass fibers are elastic and the polyamide matrix is modeled using a $\mathrm{J}_{2}$-EVP model with the linear-exponential hardening law described by Eq. (75), and the visco-plastic Perzyna's model, which reads

$$
g_{v}\left(\sigma^{\mathrm{eq}}, p\right)=\kappa\left(\frac{f}{\sigma_{\mathrm{Y}}+R(p)}\right)^{m}
$$

where $\kappa[1 / \mathrm{s}]$ is the visco-plastic modulus and $m$ is the visco-plastic exponent. The material properties of the two phases read: 
- Matrix: Elastic Young's modulus $E_{0}^{\mathrm{el}}=2.1 \mathrm{GPa}$; Poisson ratio $\nu_{0}=0.3$; yield stress $\sigma_{\mathrm{Y}_{0}}=29 \mathrm{MPa}$; first hardening coefficient $h_{1_{0}}=139 \mathrm{MPa}$; second hardening coefficient $h_{2_{0}}=32.7 \mathrm{MPa}$; hardening exponent $n_{0}=319.4$; visco-plastic modulus $\kappa_{0}=2.5 \mathrm{~s}^{-1}$; and visco-plastic exponent $m_{0}=5$.

- Inclusions: Elastic Young's modulus $E_{\mathrm{I}}^{\mathrm{el}}=72 \mathrm{GPa}$; Poisson ratio $\nu_{\mathrm{I}}=0.22$; volume fraction $v_{\mathrm{I}}=15.7 \%$; and aspect ratio of $\alpha_{\mathrm{I}}=15$.

Uniaxial tension is applied along the short fiber direction, which makes the test challenging. The short glass fiber-reinforced polyamide was studied using the FE method and a variational approach by Brassart et al. (2012).

Figure 6 compares the predictions of the incremental-secant approach with the FE results and with the predictions of the variational approach provided by Brassart et al. (2012). One more time, when considering the incremental-secant MFH with first statistical moment estimates and second statistical moment estimates, the residual stress in the matrix phase is successively considered $\left(C_{0}^{\mathrm{r}}\right.$ secant operator $)$ and neglected $\left(C_{0}^{0}\right.$ secant operator). On the one hand, the results obtained using the incremental-secant approach with first statistical moment estimates, see Fig. 6(a), are clearly over-estimating the composite material response since the yielding point is not well captured. In particular when the residual stress in the matrix is kept, the hardening phase does not exhibit the right slope. On the other hand, when considering the second statistical moment estimates, see Fig. 6(b), the solutions obtained with or without considering the residual stress in the matrix phase are in better agreement with the FE results, in particular when considering the residual. For both the variational approach and the incremental-secant approach with second statistical moment estimates, the yield point is not captured with much accuracy since the composite material involves short fibers, and the predicted composite hardening law has a lower slope than for the FE solution. Because the variational approach developed by Brassart et al. (2012) assumes a uniform polarization in the composite material, the slope of the composite hardening response is further from the FE predictions than the developed method which considers different residual strain and stress in the different phases. In the remaining of this paper we will thus only consider two cases: (i) the incremental-secant approach with first statistical moment estimates combined to the $C_{0}^{0}$ secant operator, and (ii) the incremental-secant approach with second statistical moment estimates combined to the $C_{0}^{\mathrm{r}}$ secant operator.

\subsubsection{Polymer with Prandtl's visco-plastic model reinforced with short glass fibers}

The aligned short glass fibers are elastic and the polymer matrix is modeled using a $\mathrm{J}_{2}$-EVP model with a linear-exponential hardening law (75), and with a Prandtl's model

$$
g_{\mathrm{v}}=\frac{\sigma_{\mathrm{Y}}}{\eta}\left(\sinh \left(\frac{f}{\beta}\right)\right)^{m},
$$

where $\eta$ is the visco-plastic coefficient, where $\beta$ has the unit of a stress, and where $m$ is the visco-plastic exponent. The material properties of the two phases read: 


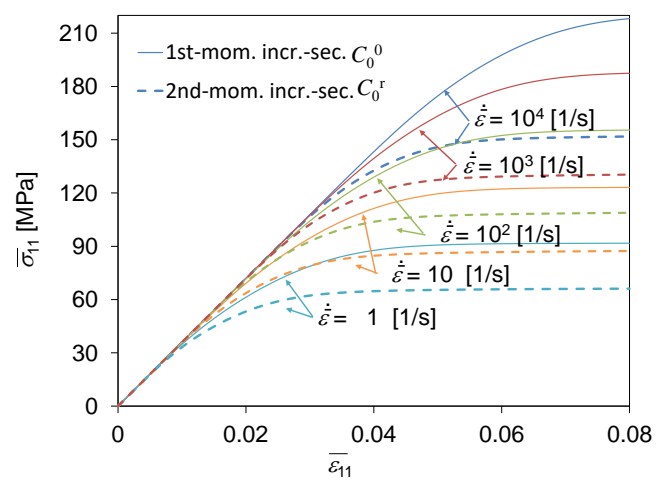

Figure 7: Short glass fiber-reinforced polymer under uniaxial loading with a matrix material following the Prandtl's visco-plastic model.

- Matrix: Elastic Young's modulus $E_{0}^{\mathrm{el}}=1066.5 \mathrm{MPa}$; Poisson ratio $\nu_{0}=0.4$; yield stress $\sigma_{\mathrm{Y}_{0}}=2 \mathrm{MPa}$; first hardening coefficient $h_{1_{0}}=1.193 \mathrm{MPa}$; second hardening coefficient $h_{2_{0}}=9.317 \mathrm{MPa}$; hardening exponent $n_{0}=107.57$; visco-plastic coefficient $\eta_{0}=7.728 \mathrm{MPa} \cdot \mathrm{s} ; \beta=10 \mathrm{MPa}$; and visco-plastic exponent $m_{0}=2.303$.

- Inclusions: Elastic Young's modulus $E_{\mathrm{I}}^{\mathrm{el}}=7.2 \times 10^{4} \mathrm{MPa}$; Poisson ratio $\nu_{\mathrm{I}}=0.22$; volume fraction $v_{\mathrm{I}}=7.1 \%$; and aspect ratio of $\alpha_{\mathrm{I}}=20$.

The composite material is loaded along the fibers direction at different strain rates, and the results are presented in Fig. 7. It can be seen that accounting for the second statistical moment estimates strongly affects the predictions.

\subsubsection{E-glass fiber reinforced Nylon}

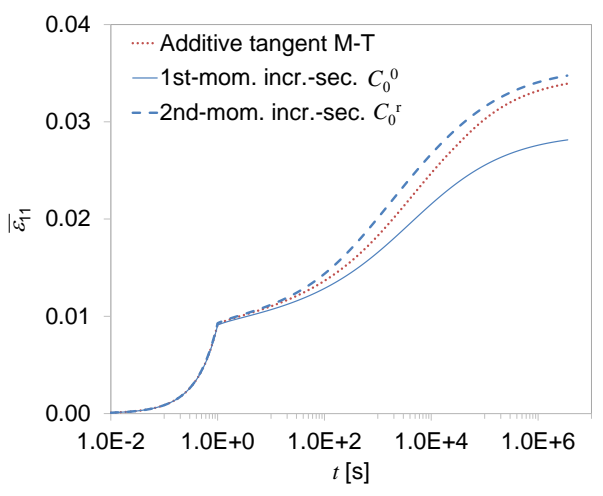

(a) Creep

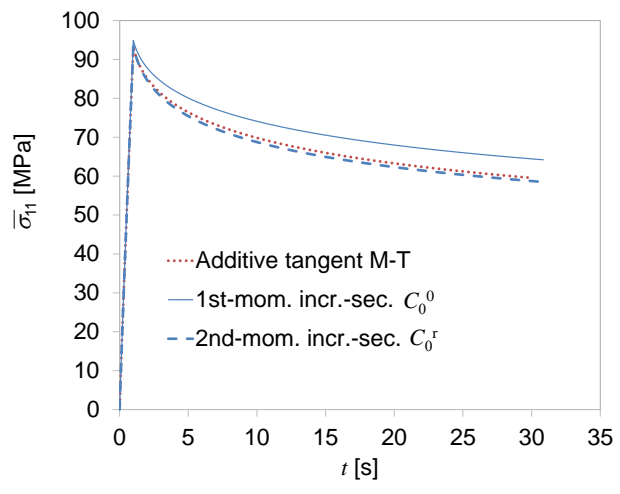

(b) Relaxation

Figure 8: E-Glass fiber reinforced Nylon response. The method predictions are compared with the prediction of the M-T additive tangent interaction law. (a) Creep test. (b) Relaxation test. 
The considered material system is E-Glass fiber reinforced Nylon. The E-glass fibers are assumed to follow an elastic behavior. The Nylon matrix is modeled using a $\mathrm{J}_{2}$-EVP model with the linear-exponential hardening law described by Eq. (75) and the viscoplastic Perzyna's model described by Eq. (76). The mechanical properties of the two phases are:

- Matrix: Elastic Young's modulus $E_{0}^{\mathrm{el}}=1.2 \mathrm{GPa}$; Poisson ratio $\nu_{0}=0.4$; yield stress $\sigma_{\mathrm{Y}_{0}}=1.5 \mathrm{MPa}$; first hardening coefficient $h_{1_{0}}=240 \mathrm{MPa}$; second hardening coefficient $h_{2_{0}}=4 \mathrm{MPa}$; hardening exponent $n_{0}=300$; visco-plastic modulus $\kappa_{0}=\frac{\sigma_{\mathrm{Y}_{0}}}{\eta}$, with $\eta=8.0 \times 10^{4} \mathrm{MPa} \cdot \mathrm{s}$; and visco-plastic exponent $m_{0}=3$.

- Inclusions: Elastic Young's modulus $E_{\mathrm{I}}^{\mathrm{el}}=72 \mathrm{GPa}$; Poisson ratio $\nu_{\mathrm{I}}=0.22$; volume fraction $v_{\mathrm{I}}=17.49 \%$; and aspect ratio of $\alpha_{\mathrm{I}}=6$.

The composite is loaded along the fiber longitudinal direction, and creep and relaxation tests are carried out, successively:

Creep: The composite is loaded to a given peak stress $\sigma_{\text {peak }}=30.0 \mathrm{MPa}$ in 1.0 second. The creep process is simulated afterwards under this constant peak stress.

Relaxation: The composite is loaded to reach a peak strain of 0.03 in $1.0 \mathrm{~s}$. The relaxation process is simulated afterwards under this constant peak strain.

Figure 8 compares the predictions of the incremental-secant approach with the results obtained using the additive tangent Mori-Tanaka (M-T) interaction law, see the review by Mercier et al. (2012). Both the incremental secant approach and the additive interaction law method solve the nonlinear MFH in an incremental way. On the one hand the additive interaction law splits the increment of strain into elastic and anelastic parts, and carries out the homogenization on the elastic and anelastic strain increments, using repectively the elastic moduli and the tangent moduli of the phase. On the other hand, the incremental secant process takes into account the increment of the anelastic strain and the total elastic strain altogether during the homogenization through the evaluation of the incremental secant moduli. Both methods give a good prediction of the composite macro response. However, since the tangent moduli of the additive interaction law are computed based on the average strain in the phase, which corresponds to a first statistical moment estimates of the strain field, it is expected to provide a stiffer prediction than when considering second statistical moment estimates as in the incremental secant approach. It can be seen in Fig. 8 that the predictions using the second statistical moment estimates are in good agreement with the IL, for both the creep, see Fig. 8(a), and relaxation, see Fig. 8(b), tests, although the IL predicts slightly stiffer results, as expected.

\subsection{Composites with two elasto-visco-plastic phases}

Both phases of the composite material exhibit an EVP behavior. The hardening function of each phase follows a power-law which is expressed by

$$
R(p)=k p^{n},
$$

where $k$ is the hardening modulus and $n$ the hardening exponent. The overstress due to the rate-dependency is described by the Perzyna's visco-plastic model (76). 


\subsubsection{Study of the loading condition effects}

The material parameters of the considered composite material are

- Matrix: Elastic Young's modulus $E_{0}^{\mathrm{el}}=70 \mathrm{GPa}$; Poisson ratio $\nu_{0}=0.33$; yield stress $\sigma_{\mathrm{Y}_{0}}=70 \mathrm{MPa}$; hardening coefficient $k_{0}=4 \mathrm{GPa}$; hardening exponent $n_{0}=$ 0.4 ; visco-plastic modulus $\kappa_{0}=3 \times 10^{-4} \mathrm{~s}^{-1}$; and visco-plastic exponent $m_{0}=1.5$.

- Inclusions: Elastic Young's modulus $E_{\mathrm{I}}^{\mathrm{el}}=400 \mathrm{GPa}$; Poisson ratio $\nu_{\mathrm{I}}=0.286$; yield stress $\sigma_{\mathrm{Y}_{\mathrm{I}}}=400 \mathrm{MPa}$; hardening coefficient $k_{\mathrm{I}}=8 \mathrm{GPa}$; hardening exponent $n_{\mathrm{I}}=0.4$; visco-plastic modulus $\kappa_{\mathrm{I}}=2 \times 10^{-4} \mathrm{~s}^{-1}$; and visco-plastic exponent $m_{\mathrm{I}}=1.5$.

The spherical EVP particles have a volume fraction $v_{\text {I }}$ of either $15 \%$ or $30 \%$. Various loading conditions are considered. We study the influence of strain rate, triaxiality, and monotonic vs. cyclic loading conditions. For the different loading cases, the results obtained using the incremental-secant approach with first statistical moment estimates and with second statistical moment estimates are compared to the finite element (FE) simulations provided by Pierard et al. (2007); Doghri et al. (2010).

Different monotonic loading conditions corresponding to different triaxiality states are first considered:

Loading \#1: Uniaxial loading at different strain rates.

Loading \#2: In-plane shearing at different shear strain rates $\dot{\bar{\varepsilon}}_{12}$.

Loading \#3: In-plane bi-axial loading at different strain rates $\dot{\bar{\varepsilon}}_{11}=\dot{\bar{\varepsilon}}_{22}$.

Figure 9 shows that the predictions using the incremental-secant approach with second statistical moment estimates perfectly match the FE results, while the results obtained when considering the first statistical moment slightly underestimate the composite response.

A uniaxial cyclic loading is then performed at two different strain rates, $\dot{\bar{\varepsilon}}_{11}=$ $\pm 10^{-3} \mathrm{~s}^{-1}$ and $\dot{\bar{\varepsilon}}_{11}= \pm 10^{-6} \mathrm{~s}^{-1}$ with the composite material with an inclusions volume fraction $v_{\text {I }}=30 \%$. Figure 10(a) compares the composite response with the FE predictions. Both incremental secant MFH approaches with first and second statistical moment estimates are in good agreement with the FE results Pierard et al. (2007); Doghri et al. (2010). The effect of the time increment $\Delta t$ on the predicted composite material response is studied in Fig. 10(b) for the incremental secant MFH approach with second statistical moment estimates. The figure shows that the solution has already converged when considering 80 time steps to study the cyclic loading. The von Mises stress evolution in the two phases are compared to the FE predictions Pierard et al. (2007); Doghri et al. (2010) in 10(c), where it can be seen that the incremental-secant approach underestimates slightly the FE predictions. Finally, the equivalent plastic strains reached at the end of the uniaxial tension-compression cycle in the different phases are illustrated in 


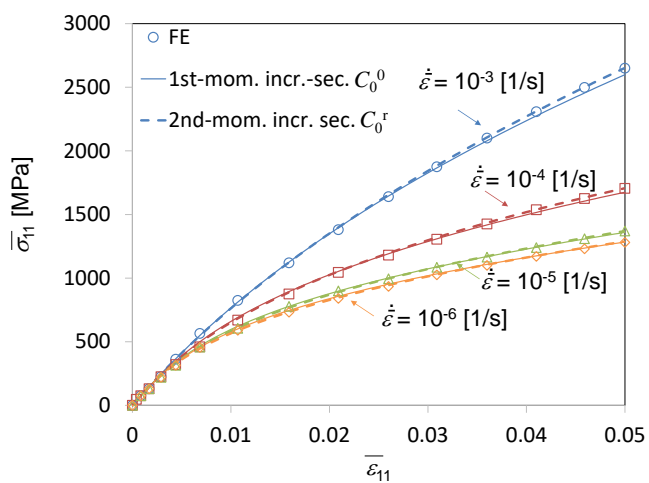

(a) $v_{\mathrm{I}}=15 \%$, uniaxial loading

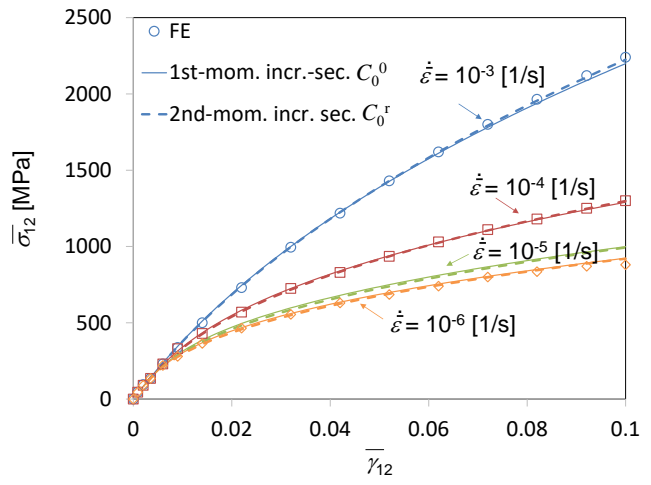

(c) $v_{\mathrm{I}}=30 \%$, shear loading

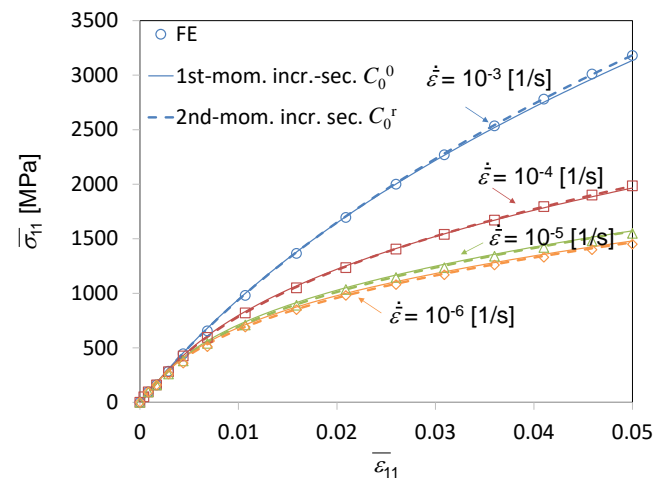

(b) $v_{\mathrm{I}}=30 \%$, uniaxial loading

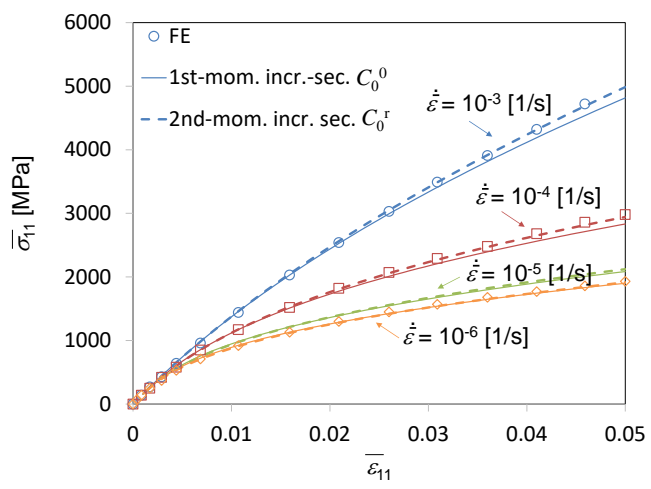

(d) $v_{\mathrm{I}}=30 \%$, biaxial loading

Figure 9: Composite material with two EVP phases under monotonic loading conditions. The method predictions for different strain rates are compared with the FE results provided by Doghri et al. (2010). (a) Uniaxial loading with an inclusions volume fraction $v_{\mathrm{I}}=15 \%$. (b) Uniaxial loading with an inclusions volume fraction $v_{\mathrm{I}}=30 \%$. (b) Shear loading with an inclusions volume fraction $v_{\mathrm{I}}=30 \%$. (d) Biaxial loading with an inclusions volume fraction $v_{\mathrm{I}}=30 \%$.

Fig. 10(d). The cumulative distributions were extracted from the FE results in Pierard (2006). The incremental-secant MFH approaches predict equivalent plastic strains which fall below the median values of their respective distributions. When using the second statistical moment estimates, the predicted equivalent plastic strain is larger than when considering the first statistical moment estimates only, in particular in the matrix phase.

\subsubsection{Study of the phases response}

The material parameters of the considered composite material are

- Matrix: Elastic Young's modulus $E_{0}^{\mathrm{el}}=100 \mathrm{GPa}$; Poisson ratio $\nu_{0}=0.3$; yield stress $\sigma_{\mathrm{Y}_{0}}=100 \mathrm{MPa}$; hardening coefficient $k_{0}=5 \mathrm{GPa}$; hardening exponent $n_{0}=1$; visco-plastic modulus $\kappa_{0}=3 \times 10^{-4} \mathrm{~s}^{-1}$; and visco-plastic exponent $m_{0}=$ 10.

- Inclusions: Elastic Young's modulus $E_{\mathrm{I}}^{\mathrm{el}}=500 \mathrm{GPa}$; Poisson ratio $\nu_{\mathrm{I}}=0.3$; yield 


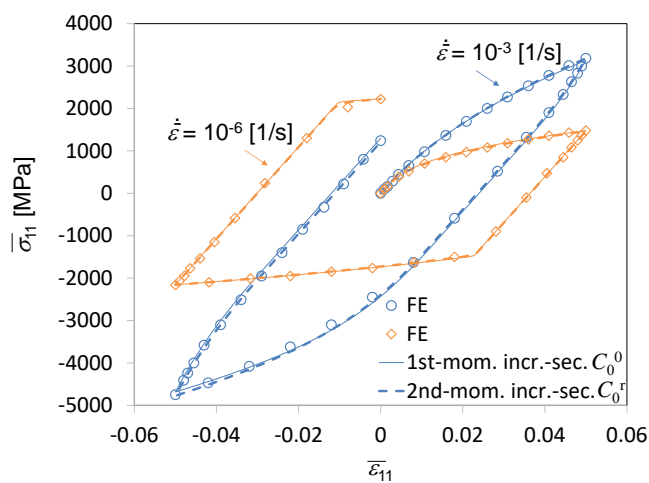

(a) Composite response

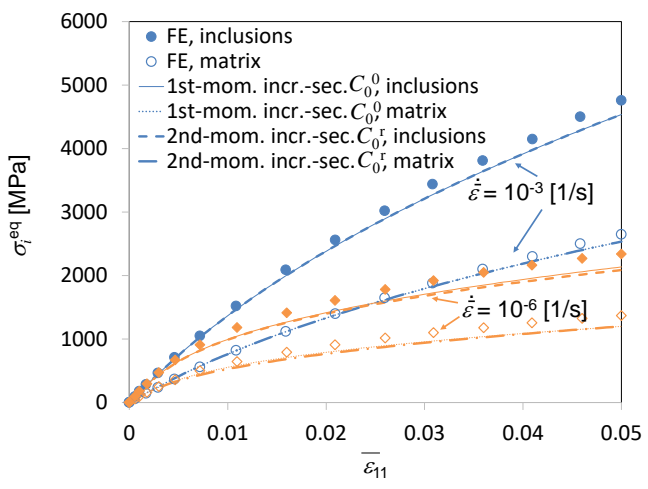

(c) Phases response

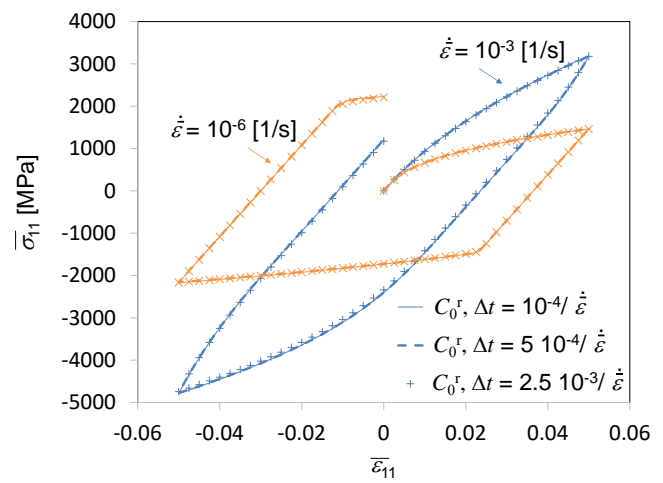

(b) Effect of time step

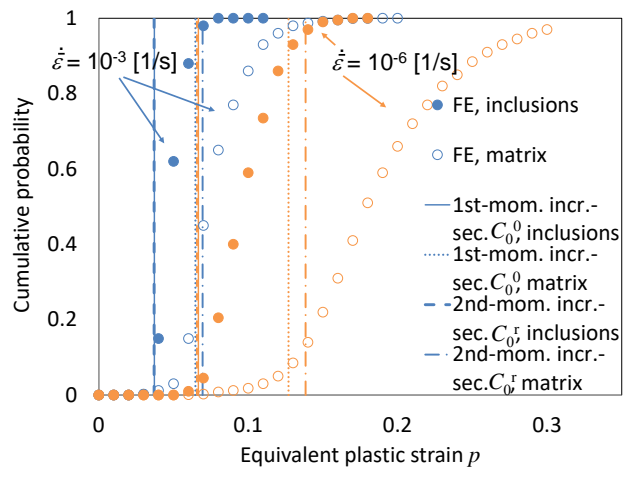

(d) Equivalent plastic strain

Figure 10: Composite material with two EVP phases under uniaxial cyclic loading with an inclusions volume fraction of $v_{\mathrm{I}}=30 \%$. The method predictions for different strain rates are compared with the FE results provided by Pierard (2006); Pierard et al. (2007); Doghri et al. (2010). (a) Composite response. (b) Effect of the time increment $\Delta t$ on the composite response with the $C_{0}^{\mathrm{r}}$ incremental-secant approach with second statistical moment estimates. (c) Phases von Mises stress evolution histories. (d) Equivalent plastic strain distribution at the end of the tension-compression cycle.

stress $\sigma_{\mathrm{Y}_{\mathrm{I}}}=500 \mathrm{MPa}$; hardening coefficient $k_{\mathrm{I}}=5 \mathrm{GPa}$; hardening exponent $n_{\mathrm{I}}=$ 1.; visco-plastic modulus $\kappa_{\mathrm{I}}=3 \times 10^{-4} \mathrm{~s}^{-1}$; and visco-plastic exponent $m_{\mathrm{I}}=10$.

The spherical EVP particles have a volume fraction $v_{\text {I }}$ of either $10 \%$ or $25 \%$ and a uniaxial cyclic loading is performed with a strain rate of $\dot{\bar{\varepsilon}}_{11}= \pm 10^{-3} \mathrm{~s}^{-1}$. The results obtained using the incremental-secant approach with first statistical moment estimates and with second statistical moment estimates are compared to the finite element (FE) simulation results and to the predictions of the additive tangent Mori-Tanaka (M-T) interaction law, both provided by Czarnota et al. (2015).

Besides the composite response, the phases responses are also analyzed in Fig. 11. It can be seen in Figs. 11(a) and 11(b) that the predictions using the incrementalsecant approach with second statistical moment estimates are in good agreement with 


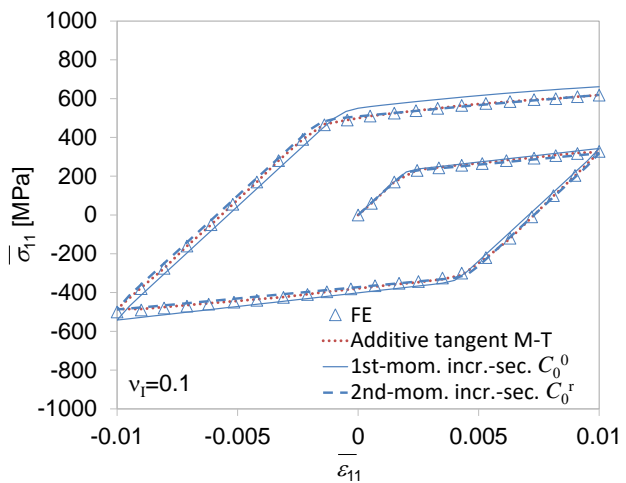

(a) $v_{\mathrm{I}}=10 \%$, composite response

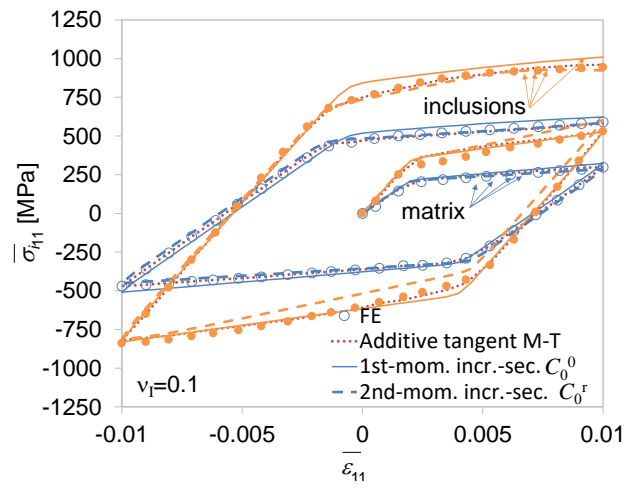

(c) $v_{\mathrm{I}}=10 \%$, phases stress vs. composite strain

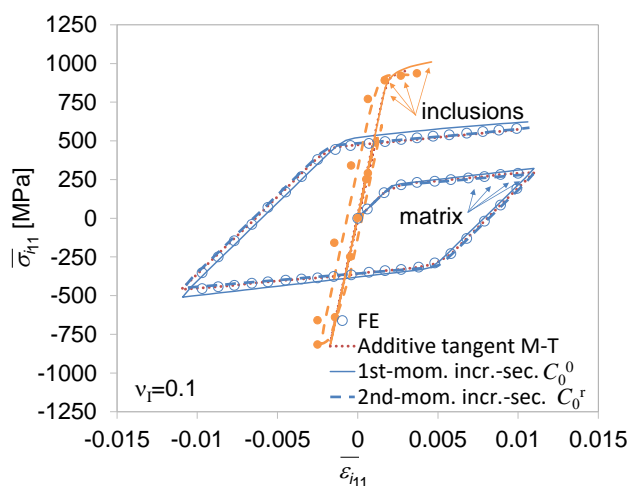

(e) $v_{\mathrm{I}}=10 \%$, phases stress vs. phases strain

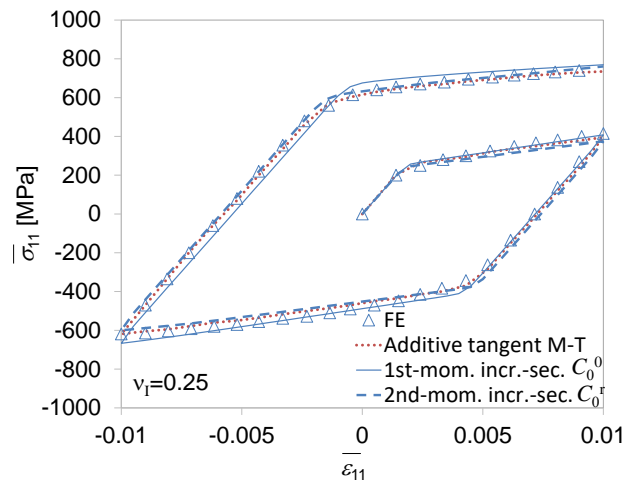

(b) $v_{\mathrm{I}}=25 \%$, composite response

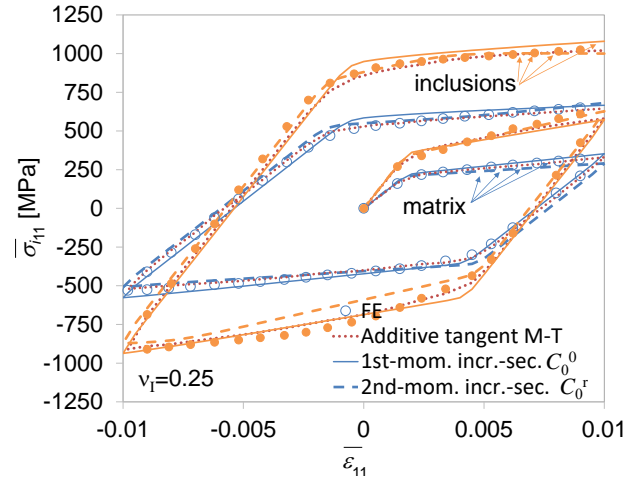

(d) $v_{\mathrm{I}}=25 \%$, phases stress $v s$. composite strain

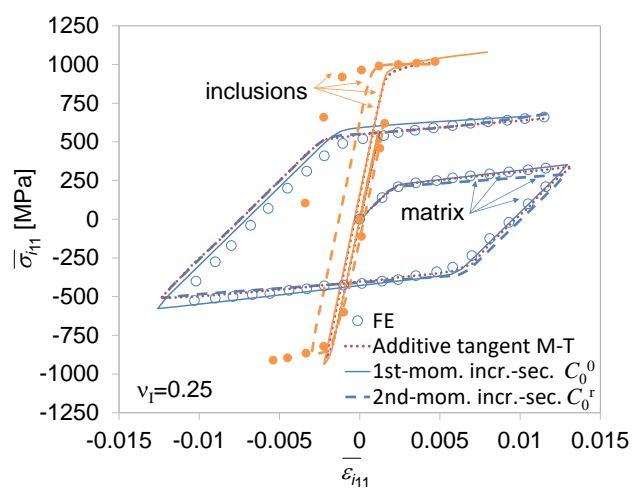

(f) $v_{\mathrm{I}}=25 \%$, phases stress vs. phases strain

Figure 11: Composite material with two EVP phases under monotonic loading conditions. The method predictions are compared with the FE results and the predictions of the additive tangent Mori-Tanaka (M-T) interaction law, both provided by Czarnota et al. (2015). (a, b) Homogenized material response; (c-d) Phases responses in terms of the composite material strain; (e-f) Phases responses in terms of the phases averaged strain. Left column corresponds to inclusions volume fraction $v_{\mathrm{I}}=10 \%$, and right column to inclusions volume fraction $v_{\mathrm{I}}=25 \%$. 
the FE results and are of comparable accuracy with the additive tangent interaction law, while the results obtained when considering the first statistical moment exhibit a slight discrepancy. A similar conclusion can be drawn when comparing the phases response in terms of the composite homogenized strain in Figs. 11(c) and 11(d). Finally the phases averaged stress evolutions in terms of their averaged strain are illustrated in Figs. 11(e) and 11(f) in which it can be seen that the incremental-secant approach with second statistical moment estimates improves the prediction of the strain contrast as compared with the other methods, although for the higher inclusions volume fraction the agreement with the FE results is not as good.

\subsection{Experimental validation: spherical SiC particle reinforced aluminum matrix}

The experimental data reported by Li and Weng (1998) for the study of a spherical $\mathrm{SiC}$ particle reinforced aluminum matrix are exploited in this section in order to validate the method.

The SiC spherical particles are assumed to follow an elastic behavior. The aluminum matrix is modeled using a $\mathrm{J}_{2}$-EVP model with an exponential isotropic hardening function which reads

$$
R(p)=k[1-\exp (-n p)],
$$

and with a power-law visco-plastic function

$$
g_{v}\left(\sigma^{\mathrm{eq}}, p\right)=\kappa\left(\frac{\sigma^{\mathrm{eq}}}{\sigma_{\mathrm{Y}}+R(p)}\right)^{m} .
$$

The material properties of the two phases have been identified by Doghri et al. (2010) and read:

- Matrix: Elastic Young's modulus $E_{0}^{\mathrm{el}}=70 \mathrm{GPa}$; Poisson ratio $\nu_{0}=0.33$; yield stress $\sigma_{\mathrm{Y}_{0}}=60 \mathrm{MPa}$; hardening coefficient $k_{0}=40 \mathrm{MPa}$; hardening exponent $n_{0}=54.9 ;$ visco-plastic modulus $\kappa_{0}=4.4 \times 10^{-6} \mathrm{~s}^{-1}$; and visco-plastic exponent $m_{0}=4.61$.

- Inclusions: Elastic Young's modulus $E_{\mathrm{I}}^{\mathrm{el}}=490 \mathrm{GPa}$; Poisson ratio $\nu_{\mathrm{I}}=0.17$; and volume fraction $v_{\mathrm{I}}=10 \%$.

Uniaxial tension loading were applied by $\mathrm{Li}$ and Weng (1998) on both the unreinforced matrix and on the composite material at a strain rate of $\pm 10^{-3} \mathrm{~s}^{-1}$, and the results are reported in Fig. 12. The matrix response obtained with the reported material parameters is also reported, justifying the considered values. Finally, this figure also shows that the predictions obtained with the incremental-secant approach when considering the second statistical moment estimates are in perfect agreement with the experimental results, although the results obtained when considering the first statistical moment slightly overestimate the composite response. 


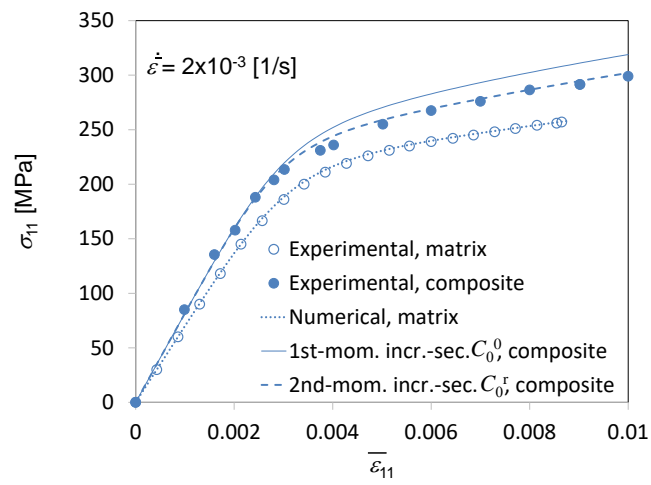

Figure 12: Spherical SiC particle reinforced aluminum matrix under uniaxial loading with an inclusions volume fraction of $v_{\mathrm{I}}=10 \%$. The method predictions for a given strain rate are compared with the experimental results provided by Li and Weng (1998).

\subsection{Inelastic solid with spherical elastic inclusions}

In this part, a visco-plastic material has neither yielding stress nor plastic hardening effect and its non-linear "visco-plastic" law is obtained from a dissipation potential, which takes the form of the power-law:

$$
\phi(\dot{p})=\frac{\sigma_{\mathrm{Y}} \dot{\varepsilon}_{0}}{1+m}\left(\frac{\dot{p}}{\dot{\varepsilon}_{0}}\right)^{1+m},
$$

where $\dot{\varepsilon}_{0}, \sigma_{\mathrm{Y}}$, and $m$ are material constants. From Eq. (81), the von Mises stress follows

$$
\sigma^{\mathrm{eq}}=\frac{\partial \phi(\dot{p})}{\partial \dot{p}}=\sigma_{\mathrm{Y}}\left(\frac{\dot{p}}{\dot{\varepsilon}_{0}}\right)^{m}
$$

and the visco-plastic law of the matrix reads

$$
\dot{p}=g_{v}\left(\sigma^{\mathrm{eq}}, p\right)=\dot{\varepsilon}_{0}\left(\frac{\sigma^{\mathrm{eq}}}{\sigma_{\mathrm{Y}}}\right)^{\frac{1}{m}} .
$$

For this kind of material behavior for which the yield stress is zero, only hard inclusions are considered. The case of soft inclusions is discussed in Appendix B.

\subsubsection{Monotonic loading}

Spherical isotropic linear elastic inclusions are embedded in a non-linear "viscoplastic" matrix. The matrix follows the visco-plastic law (83), and the properties of the material system first studied by Lahellec and Suquet (2007a) for $m=0.2$ and then extended by Czarnota et al. (2015) to other values of $m$, with

- Matrix: Elastic Young's modulus $E_{0}^{\mathrm{el}}=70 \mathrm{GPa}$; Poisson ratio $\nu_{0}=0.3 ; \dot{\varepsilon}_{0_{0}}=$ $10^{-2} \mathrm{~s}^{-1} ; \sigma_{\mathrm{Y}_{0}}=480 \mathrm{MPa}$; and $m_{0} \in\left[\begin{array}{ll}0.01 & 1\end{array}\right]$.

- Inclusions: Elastic Young's modulus $E_{\mathrm{I}}^{\mathrm{el}}=400 \mathrm{GPa}$; Poisson ratio $\nu_{\mathrm{I}}=0.2$; and volume fraction $v_{\mathrm{I}}=25 \%$. 


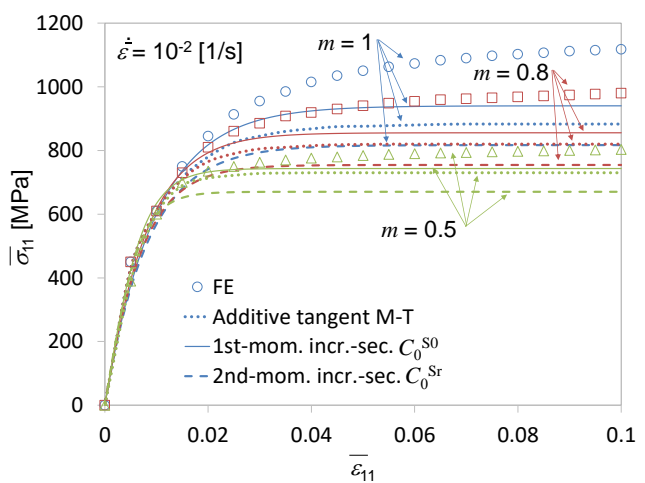

(a) $m_{0} \geq 0.5$

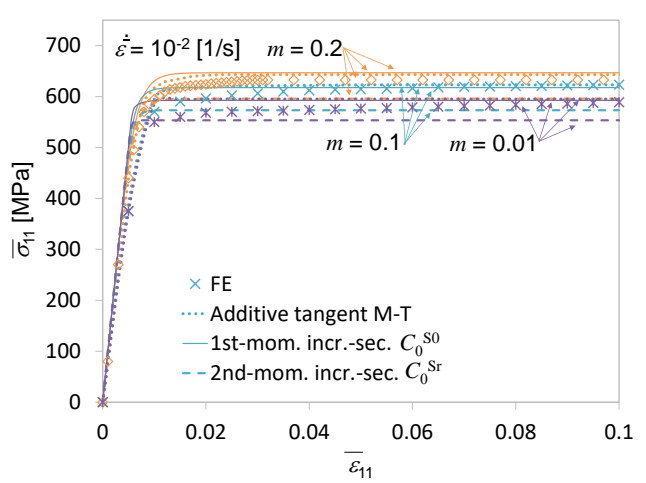

(b) $m_{0}<0.5$

Figure 13: Inelastic solid with spherical elastic inclusions under monotonic loading. The method predictions for different values of $m_{0}$ are compared with the prediction of the M-T additive tangent interaction law and with the FE results, both provided by Czarnota et al. (2015).

An uniaxial tension is performed at a strain rate of $10^{-2} \mathrm{~s}^{-1}$. The results obtained by the developed method are compared in Fig. 13 with the finite element results from the work of Czarnota et al. (2015) and to the M-T additive tangent interaction law predictions reported Mercier et al. (2012). For values of $m_{0}$ higher than 0.5, see Fig. 13(a), the different homogenization methods under-predict the saturation stage. Following the discussion by Czarnota et al. (2015), these cases are really difficult to model due to the large contrast in phases behavior, including with finite element methods for which an axisymmetric unit cell and an RVE with 30 inclusions yield different predictions. The accuracy of the homogenization methods is better for values of $m_{0}$ lower than 0.5 , see Fig. 13(b). For these latter cases, the incremental-secant approach with first statistical moment estimates predict results similar to the M-T additive tangent interaction law and both methods slightly overestimate the FE results in the pre-saturation stage. The results obtained using the incremental-secant approach with the second statistical moment estimates underestimate the FE results.

\subsubsection{Non-proportional loading}

The considered composite material is an EVP matrix reinforced by elastic inclusions with isotropic dispersion as described by Idiart and Lahellec (2016). Both phases are isotropic and incompressible, which means that the two bulk moduli $\kappa_{0} \rightarrow \infty$ and $\kappa_{\mathrm{I}} \rightarrow$ $\infty$.

The matrix follows the visco-plastic law (83), and the properties of the material system reported by Idiart and Lahellec (2016) are

- Matrix: Elastic shear modulus $\mu_{0}^{\mathrm{el}}=10^{3} \cdot \sigma_{\mathrm{Y}_{0}} ; \dot{\varepsilon}_{0_{0}}=10^{-3} \mathrm{~s}^{-1}$; and $m_{0}=0.2$.

- Inclusions: Elastic shear modulus $\mu_{\mathrm{I}}^{\mathrm{el}}=R \mu_{0}^{\mathrm{el}}$ with $R=5$; and volume fraction $v_{\mathrm{I}}=30 \%$. 


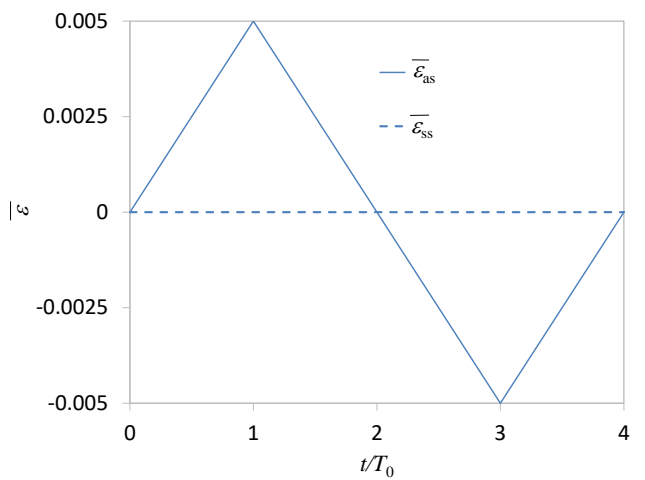

(a) Loading \#1

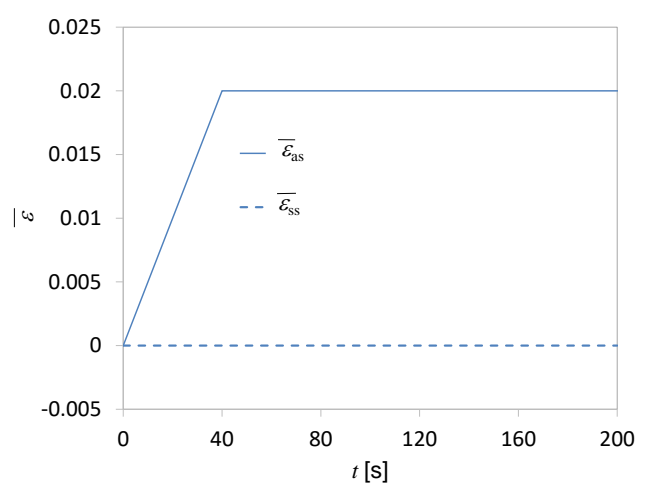

(c) Loading \#2

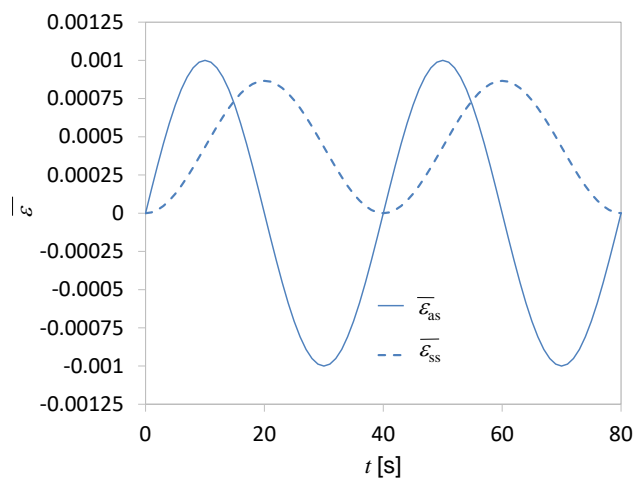

(e) Loading \#3

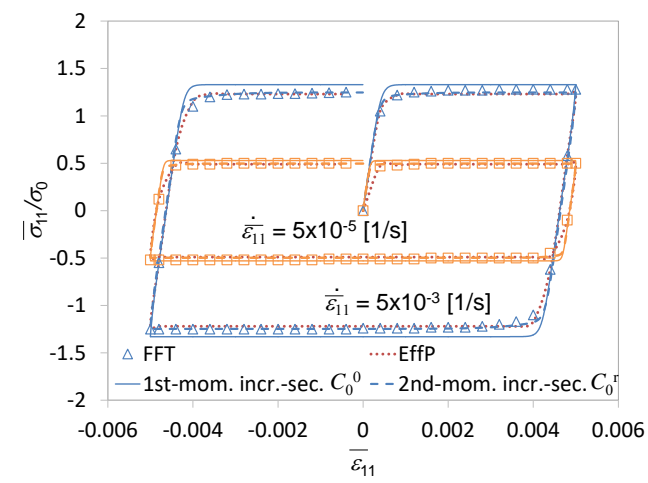

(b) Loading $\# 1, R=5$

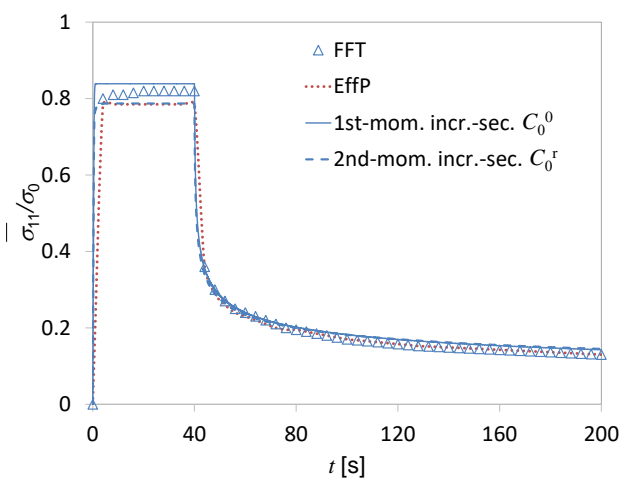

(d) Loading \#2, $R=5$

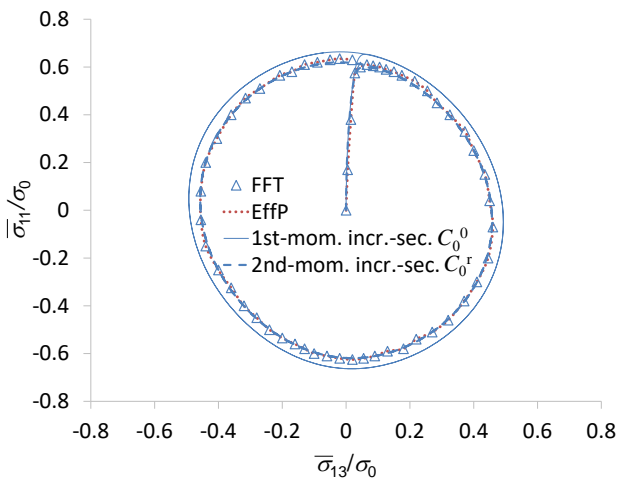

(f) Loading \#3, $R=5$

Figure 14: Composite response of the anelastic solid with spherical elastic inclusions for the case $R=5$. The method predictions are compared with the FFT results and the variational method predictions obtained using "effective polarization (EffP)" estimates provided by Idiart and Lahellec (2016). (a-b) Axisymmetric shear loading cycles at two different strain rates. (c-d) Axisymmetric shear loading ramp. (e-f) Rotating deformation.

The material is initially free of any internal stress, and then, subjected to a strain 
function in the form of

$$
\bar{\varepsilon}(t)=\bar{\varepsilon}_{s s}(t)\left(\mathbf{e}_{1} \otimes \mathbf{e}_{3}+\mathbf{e}_{3} \otimes \mathbf{e}_{1}\right)+\bar{\varepsilon}_{a s}(t)\left(\mathbf{e}_{1} \otimes \mathbf{e}_{1}-\frac{1}{2} \mathbf{e}_{2} \otimes \mathbf{e}_{2}-\frac{1}{2} \mathbf{e}_{3} \otimes \mathbf{e}_{3}\right),
$$

where $\left\{\mathbf{e}_{i}\right\}$ defines the orthonormal basis. Three loading cases are studied, respectively,

Loading \#1 Radial deformation consisting of a triangular axisymmetric shear, see Fig. 14(a):

$$
\bar{\varepsilon}_{s s}(t)=0 \text { and } \bar{\varepsilon}_{a s}(t)=\dot{r}\left(t-2 T_{0}\left\lfloor\frac{t}{2 T_{0}}+\frac{1}{2}\right\rfloor\right)(-1)^{\left\lfloor\frac{t}{2 T_{0}}+\frac{1}{2}\right\rfloor},
$$

where $\lfloor\cdot\rfloor$ denotes the floor function. Two strain rates are considered: $\dot{r}=5 \times$ $10^{-5} \mathrm{~s}^{-1}$, with $T_{0}=100 \mathrm{~s}, t \in[0,400] \mathrm{s} ;$ and $\dot{r}=5 \times 10^{-3} \mathrm{~s}^{-1}$, with $T_{0}=1 \mathrm{~s}$, $t \in[0,4] \mathrm{s}$.

Loading \#2: Radial deformation consisting of an axisymmetric loading ramp up to a constant value, see Fig. 14(c):

$$
\bar{\varepsilon}_{s s}(t)=0 \text { and } \bar{\varepsilon}_{a s}(t)=\left\{\begin{array}{ll}
\dot{r} t & 0 \leq t \leq T_{0} \\
\dot{r} T_{0} & T_{0}<t
\end{array},\right.
$$

where $\dot{r}=5 \times 10^{-4} \mathrm{~s}^{-1}$, and $T_{0}=40 \mathrm{~s}$.

Loading \#3: Rotating deformation, see Fig. 14(e):

$$
\bar{\varepsilon}_{s s}(t)=\frac{\sqrt{3}}{4} r[1-\cos (\omega t)] \text { and } \bar{\varepsilon}_{a s}(t)=r \sin (\omega t),
$$

where $r=10^{-3}$ and $\omega=\pi / 20 \mathrm{rad} / \mathrm{s}$.

The results obtained using the incremental-secant approach with first statistical moment estimates and with second statistical moment estimates are reported in Fig. 14 for $R=5$. The method predictions are compared with the results obtained with the FFT method and the variational method using "effective polarization (EffP)" estimates provided by Idiart and Lahellec (2016). It can be seen that while the incremental-secant method slightly overestimates the results when using the first statistical moment estimates, and is of comparable accuracy with the variational method when considering the second statistical moment estimates.

\section{Conclusions}

In this paper, the incremental-secant MFH method previously developed for rateindependent EP and non-local-damage-enhanced EP behaviors is extended to rate-dependent EVP material behaviors. In the incremental-secant formulation, a virtual elastic unloading is performed at the composite level in order to evaluate the residual stress and strain states in the different phases. The LCC is then defined from these states to apply a secant MFH formulation.

In particular, we have used a new second statistical moment estimate of the von Mises stress, yielding accurate results while keeping the residual stress in the matrix phase 
reached upon the virtual unloading, while the method which only considers first statistical moment estimates requires this residual stress to be canceled to predict accurate results.

Moreover, it was shown that the basis of the incremental-secant method does not require to be changed to account for the visco-plastic behaviors, and that all the modifications are limited to the constitutive material boxes of the material library. As a result the increment-secant MFH for EVP materials inherits from its intrinsic advantages which are (i) the ability to handle non-radial loading conditions, (ii) the isotropic nature of the LCC instantaneous stiffness tensor, which renders obsolete the isotropisation step of some other MFH methods, and (iii) a straightforward implementation as the homogenization method directly calls the material constitutive material boxes of an existing materials library.

Finally, in order to assess the accuracy of the method, its predictions were compared to finite element (FE) results, to Fast-Fourier-Transform (FFT) results, to experiments, and to other homogenization predictions for monotonic, cyclic, and non-radial loading conditions at different strain rates, and also for different loading conditions corresponding to different triaxiality states. It was shown that even in the challenging case of short glass fiber reinforced polymer matrix, the incremental-secant method with second statistical moment estimates predicts good to excellent results with respect to full-field $\mathrm{FE}$ and FFT results.

However, the issue of the residual stress in the matrix remains to be analyzed carefully. On the one hand, when considering the incremental-secant MFH with first statistical moment estimates, the choice of the incremental-secant moduli in the matrix phase, $C_{0}^{0}$ or $C_{0}^{\mathrm{r}}$ is governed by the relative positions of the stress and residual stress tensors with respect to the stress space origin (zero-stress state): (i) The origin lies between the stress and residual stress tensors (for a uniaxial tension this corresponds to a positive stress and a negative residual stress), which is typically the case for elastic inclusions and for composite materials made of two elasto-(visco)plastic phases for which the inclusions phase is stiffer than the matrix phase during the plastic flow. In that case, predictions are unacceptable unless the residual stress is removed in the matrix phase. (ii) Both stress and residual stress tensors lie on the same side with respect to the origin (for a uniaxial tension this corresponds to a positive stress and a positive residual stress), which is the case for composite materials made of two elasto-(visco)plastic phases for which the inclusion phase is softer than the matrix phase during the plastic flow. In that case, the residual stress should be kept in the matrix phase. This behavior was discussed in the case of elasto-plastic composites by Wu et al. (2013b). On the other hand, we have proposed a proper second statistical moment estimate of the von Mises stress which prevents removing the residual stress in the matrix phase to predict accurate results. However, this proper second statistical moment estimate of the von Mises stress also depends on the relative positions of the stress and residual stress tensors with respect to the stress space origin. (i) In the case of elastic inclusions and for composite materials made of two elasto-(visco)plastic phases for which the inclusions phase is stiffer than the matrix phase during the plastic flow, the estimate (43) in which only the equivalent stress increment is considered yields accurate prediction. However, for perfectly plastic matrix behavior the use of second statistical moments could lead to an artificial softening as discussed by $\mathrm{Wu}$ et al. (2015a) (ii) In the case of composite materials made of two elasto-(visco)plastic phases for which the inclusion phase is softer than the matrix phase during the visco-plastic flow, using the estimate (43) predicts a composite response too 
stiff, and the accuracy can be improved by using the second statistical estimate of the von Mises stress (B.2) in which the residual stress in accounted for. Note that for the case of such composite with more compliant inclusions, since the Mori-Tanaka method implies a uniform stress distribution in the inclusions phase, the use of an incremental-secant MFH with first statistical moment estimates is justified.

\section{Acknowledgment}

The research has been funded by the Walloon Region under the agreement no 1410246STOMMMAC (CT-INT 2013-03-28) in the context of the M-ERA.NET Joint Call 2014.

\section{Appendix A. Derivatives}

Appendix A.1. Derivatives of the incremental-secant operators

The derivatives of $\hat{\mathbb{C}}_{i n+1}^{\mathrm{S}}$ with respect to $\bar{\varepsilon}_{i n+1}^{\mathrm{r}}$ and with respect to $\bar{\varepsilon}_{n+1}^{\mathrm{r}}$ were obtained by Wu et al. (2015a) as

$$
\frac{\partial \hat{\mathbb{C}}_{i n+1}^{\mathrm{S}}}{\partial \bar{\varepsilon}_{i n+1}^{\mathrm{r}}}=-\frac{2 \mu_{i}^{\mathrm{el}}}{\Delta \hat{\hat{\varepsilon}}_{i n+1}^{\mathrm{r}}{ }^{\mathrm{eq}}} \mathbb{I}^{\mathrm{dev}} \otimes \frac{\partial \Delta \hat{p}_{i}}{\partial \bar{\varepsilon}_{i}^{\mathrm{r}}}
$$

with $\Delta \hat{\hat{\varepsilon}}_{i n+1}^{\mathrm{r}}{ }_{n+1}^{\mathrm{eq}}=\sqrt{\frac{2}{3} \mathbb{I d e v}^{\mathrm{de}}:\left\langle\Delta \varepsilon_{n+1}^{\mathrm{r}} \otimes \Delta \varepsilon_{n+1}^{\mathrm{r}}\right\rangle_{\omega_{i}}}$ computed from $3 \mu_{i}^{\mathrm{el}} \Delta \hat{\hat{\varepsilon}}_{i}^{\mathrm{r}}{ }_{n+1}^{\mathrm{eq}}=\Delta \hat{\hat{\sigma}}_{n+1}^{\mathrm{r}}{ }_{n+1}^{\text {trial }}{ }^{\mathrm{eq}}$, and as

$$
\frac{\partial \hat{\mathbb{C}}_{i n+1}^{\mathrm{S}}}{\partial \overline{\boldsymbol{\varepsilon}}_{n+1}^{\mathrm{r}}}=2 \mu_{i}^{\mathrm{el}} \mathbb{I}^{\mathrm{dev}} \otimes\left(\frac{\Delta \hat{p}_{i}}{3 v_{i}\left(\Delta \hat{\hat{\varepsilon}}_{i n+1}^{\mathrm{r}}\right)^{\mathrm{eq}}} \frac{\partial \hat{\mathbb{C}}^{\mathrm{el}}}{\partial \mu_{i}^{\mathrm{el}}}: \Delta \bar{\varepsilon}_{n+1}^{\mathrm{r}}-\frac{1}{\Delta \hat{\hat{\varepsilon}}_{i n+1}^{\mathrm{req}}} \frac{\partial \Delta \hat{p}_{i}}{\partial \overline{\boldsymbol{\varepsilon}}^{\mathrm{r}}}\right)
$$

where $\hat{\mathbb{C}}^{\mathrm{el}}$ is given by (38).

Finally the derivatives $\frac{\partial \Delta \hat{p}_{i}}{\partial \bar{\varepsilon}_{i}^{r}}$ and $\frac{\partial \Delta \hat{p}_{i}}{\partial \bar{\varepsilon}^{r}}$ are computed from the return mapping algorithm.

\section{Appendix A.2. Jacobian evaluation}

The Jacobian of the residual function (69) is obtained as

$$
\begin{aligned}
\mathbb{J}= & \frac{\partial \boldsymbol{F}}{\partial \bar{\varepsilon}_{\mathrm{I}}}+\frac{\partial \boldsymbol{F}}{\partial \bar{\varepsilon}_{0}}:\left.\frac{\partial \bar{\varepsilon}_{0}}{\partial \bar{\varepsilon}_{\mathrm{I}}}\right|_{\Delta \bar{\varepsilon}_{n+1}^{\mathrm{r}}}=\frac{\partial \boldsymbol{F}}{\partial \bar{\varepsilon}_{\mathrm{I}}}-\frac{v_{\mathrm{I}}}{v_{0}} \frac{\partial \boldsymbol{F}}{\partial \bar{\varepsilon}_{0}} \\
= & \hat{\mathbb{C}}_{0}^{\mathrm{S}}:\left[\mathbb{I}-\mathbb{S}^{-1}\right]-\hat{\mathbb{C}}_{\mathrm{I}}^{\mathrm{S}}-\frac{\partial \hat{\mathbb{C}}_{\mathrm{I}}^{\mathrm{S}}}{\partial \varepsilon_{\mathrm{I}}}: \Delta \bar{\varepsilon}_{\mathrm{I}}^{\mathrm{r}}- \\
& \frac{v_{\mathrm{I}}}{v_{0}} \frac{\partial \hat{\mathbb{C}}_{0}^{\mathrm{S}}}{\partial \bar{\varepsilon}_{0}}:\left[\Delta \bar{\varepsilon}_{\mathrm{I}}^{\mathrm{r}}-\mathbb{S}^{-1}: \frac{\left(\Delta \bar{\varepsilon}_{\mathrm{I}}^{\mathrm{r}}-\Delta \bar{\varepsilon}^{\mathrm{r}}\right)}{v_{0}}\right]-\frac{v_{\mathrm{I}}}{v_{0}} \hat{\mathbb{C}}_{0}^{\mathrm{S}}: \mathbb{S}^{-1}- \\
& \frac{v_{\mathrm{I}}}{v_{0}^{2}} \hat{\mathbb{C}}_{0}^{\mathrm{S}} \otimes\left(\Delta \bar{\varepsilon}_{\mathrm{I}}^{\mathrm{r}}-\Delta \bar{\varepsilon}^{\mathrm{r}}\right)::\left(\mathbb{S}^{-1} \otimes \mathbb{S}^{-1}\right):: \frac{\partial \mathbb{S}}{\partial \bar{\varepsilon}_{0}} .
\end{aligned}
$$


Moreover one has

$$
\begin{aligned}
\frac{\partial \boldsymbol{F}}{\partial \bar{\varepsilon}}= & {\left[\Delta \bar{\varepsilon}_{\mathrm{I} n+1}^{\mathrm{r}}-\frac{1}{v_{0}} \mathbb{S}^{-1}:\left(\Delta \bar{\varepsilon}_{\mathrm{I} n+1}^{\mathrm{r}}-\Delta \bar{\varepsilon}_{n+1}^{\mathrm{r}}\right)\right]: \frac{\partial \hat{\mathbb{C}}_{0 n+1}^{\mathrm{S}}}{\partial \bar{\varepsilon}}+} \\
& \frac{1}{v_{0}} \hat{\mathbb{C}}_{0 n+1}^{\mathrm{S}} \otimes\left(\Delta \bar{\varepsilon}_{\mathrm{I} n+1}^{\mathrm{r}}-\Delta \bar{\varepsilon}_{n+1}^{\mathrm{r}}\right)::\left(\mathbb{S}^{-1} \otimes \mathbb{S}^{-1}\right):: \frac{\partial \mathbb{S}}{\partial \bar{\varepsilon}}+ \\
& \frac{1}{v_{0}} \hat{\mathbb{C}}_{0 n+1}^{\mathrm{S}}: \mathbb{S}^{-1}-\Delta \bar{\varepsilon}_{\mathrm{I} n+1}^{\mathrm{r}}: \frac{\partial \hat{\mathbb{C}}_{\mathrm{I} n+1}^{\mathrm{S}}}{\partial \bar{\varepsilon}} .
\end{aligned}
$$

In these equations $\frac{\partial \hat{\mathbb{C}}_{i}^{\mathrm{S}}}{\partial \overline{\boldsymbol{\varepsilon}}_{i}}$ are given in Appendix A.1, and where $\frac{\partial \mathbb{S}}{\partial \overline{\boldsymbol{\varepsilon}}_{0}}$ is given in Appendix Appendix A.3.

\section{Appendix A.3. Derivatives of the Eshelby tensor}

As the Eshelby tensor depends on the Poisson ratio of the matrix phase, one can evaluate

$$
\begin{aligned}
\frac{\partial \mathbb{S}}{\partial \Delta \bar{\varepsilon}_{0}^{\mathrm{r}}} & =\frac{\partial \mathbb{S}}{\partial \nu} \otimes\left(\frac{\partial \nu}{\partial \mu} \frac{\partial \hat{\mu}_{0}^{\mathrm{S}}}{\partial \Delta \bar{\varepsilon}_{0}^{\mathrm{r}}}\right), \\
\frac{\partial \mathbb{S}}{\partial \Delta \bar{\varepsilon}^{\mathrm{r}}} & =\frac{\partial \mathbb{S}}{\partial \nu} \otimes \frac{\partial \nu}{\partial \mu} \frac{\partial \hat{\mu}_{0}}{\partial \Delta \bar{\varepsilon}^{\mathrm{r}}}
\end{aligned}
$$

where $\frac{\partial \hat{\mu}_{0}}{\partial \Delta \bar{\varepsilon}_{0}^{\mathrm{r}}}$ and $\frac{\partial \hat{\mu}_{0}}{\partial \Delta \bar{\varepsilon}^{\mathrm{r}}}$ are obtained from Eq. (56) using the derivatives reported in Appendix A.1.

\section{Appendix B. Soft inclusions}

The von Mises stress can be written in terms of the residual increment as

$$
{\sigma_{n+1}^{\mathrm{eq}} 2}^{2}=\left(\Delta \boldsymbol{\sigma}^{\mathrm{r}}\right)^{\mathrm{eq} 2}+\left(\boldsymbol{\sigma}_{n}^{\mathrm{res}}\right)^{\mathrm{eq} 2}+3\left(\Delta \boldsymbol{\sigma}^{\mathrm{r}}\right)^{\mathrm{dev}}:\left(\boldsymbol{\sigma}_{n}^{\mathrm{res}}\right)^{\mathrm{dev}} .
$$

From equation (B.1) we can see that, if the residual stress is non negligible, the approximation (43) can lead to a inaccurate prediction in the matrix phase of the LCC. In particular:

- In the case of negligible residual stress, i.e. if $3\left|\left\langle\left(\Delta \boldsymbol{\sigma}^{\mathrm{r}}\right)^{\mathrm{dev}}:\left(\boldsymbol{\sigma}_{n}^{\mathrm{res}}\right)^{\mathrm{dev}}\right\rangle\right| \ll\left(\Delta \hat{\hat{\sigma}}^{\mathrm{r}}\right)^{\mathrm{eq}}{ }^{2}$, the evaluation of ( $\left.\hat{\boldsymbol{\sigma}}^{\text {trial }}\right)^{\text {eq }}$ using Eq. (43) corresponds to an accurate approximation.

- In the case in which $3\left|\left\langle\left(\Delta \boldsymbol{\sigma}^{\mathrm{r}}\right)^{\mathrm{dev}}:\left(\boldsymbol{\sigma}_{n}^{\mathrm{res}}\right)^{\mathrm{dev}}\right\rangle\right| \sim\left(\Delta \hat{\hat{\sigma}}^{\mathrm{r}}{ }^{\mathrm{eq}}\right)^{2}$ and in which $\left\langle\left(\Delta \boldsymbol{\sigma}^{\mathrm{r}}\right)^{\mathrm{dev}}:\left(\boldsymbol{\sigma}_{n}^{\text {res }}\right)^{\mathrm{dev}}\right\rangle<0$, the evaluation of $\left(\hat{\boldsymbol{\sigma}}^{\text {trial }}\right)^{\text {eq }}$ using Eq. (43) corresponds to an overestimation and induces a softer LCC response. However, because of the use of the approximation $(33)$, the effect of the overestimatation of $\left(\hat{\hat{\boldsymbol{\sigma}}}^{\text {trial }}\right)^{\text {eq }}$ is weakened, leading to a correct overall response of the composite material. 


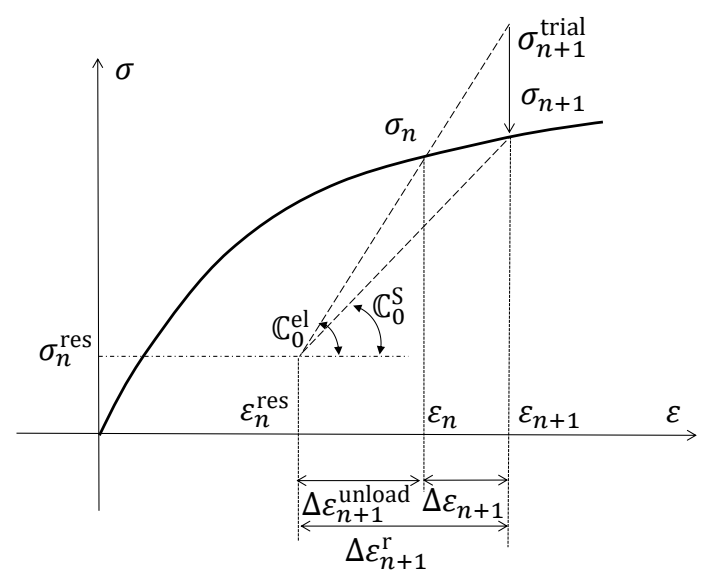

Figure B.15: Schematics of the incremental-secant formulations in the matrix phase in the context of a soft-inclusions material system

- In the other case, i.e. if $3\left|\left\langle\left(\Delta \boldsymbol{\sigma}^{\mathrm{r}}\right)^{\mathrm{dev}}:\left(\boldsymbol{\sigma}_{n}^{\mathrm{res}}\right)^{\mathrm{dev}}\right\rangle\right| \sim\left(\Delta \hat{\hat{\sigma}}^{\mathrm{r}}{ }^{\mathrm{eq}}\right)^{2}$ and $\left\langle\left(\Delta \boldsymbol{\sigma}^{\mathrm{r}}\right)^{\mathrm{dev}}:\left(\boldsymbol{\sigma}_{n}^{\text {res }}\right)^{\mathrm{dev}}\right\rangle>0$, which is usually the case for soft inclusions, as illustrated in Fig. B.15, the evaluation of $\hat{\hat{\sigma}}^{\text {trial }}{ }^{\text {eq }}$ using the approximation Eq. (43) corresponds to an the underestimated, resulting in a stiffer response of the LCC. Contrarily to the previous case, this effect is strengthened because of the approximation (33), which could lead to inaccurate predictions.

Therefore, in order to take into account the residual stress in matrix in the estimate of the von Mises stress, we use the approximation

$$
\left(\hat{\hat{\sigma}}_{n+1}^{\text {trial }}{ }^{\text {eq }}\right)^{2} \approx\left(\Delta \hat{\hat{\sigma}}^{\text {trial }}{ }^{\text {eq }}\right)^{2}+\left(\left(\overline{\boldsymbol{\sigma}}_{n}^{\text {res }}\right)^{\text {eq }}\right)^{2}+3\left(\Delta \overline{\boldsymbol{\sigma}}^{\mathrm{r}}\right)^{\text {dev }}:\left(\overline{\boldsymbol{\sigma}}_{n}^{\text {res }}\right)^{\text {dev }} .
$$

Because this approximation is a mix of first and second statistical moment estimates, it is not always accurate enough for the composites with soft inclusion.

The soft inclusion reinforced matrix material system studied by Czarnota et al. (2015) is considered here to illustrate the effect of the different assumptions of the von Mises stress estimates in the second statistical moment approach. The spherical inclusions and the matrix phase follow the elasticviscoplastic described by the power-law (83). And the material parameters for the analysis are

- Matrix: Elastic Young's modulus $E_{0}^{\mathrm{el}}=100 \mathrm{GPa}$; Poisson ratio $\nu_{0}=0.45$; yield stress $\sigma_{\mathrm{Y}_{0}}=1.0 \mathrm{GPa}$; visco-plastic reference strain rate $\dot{\varepsilon}_{0_{0}}=1.0 \mathrm{~s}^{-1}$; and viscoplastic exponent $m_{0}=0.1$.

- Inclusion: Elastic Young's modulus $E_{\mathrm{I}}^{\mathrm{el}}=100 \mathrm{GPa}$; Poisson ratio $\nu_{\mathrm{I}}=0.45$; yield stress $\sigma_{\mathrm{Y}_{\mathrm{I}}}=0.2 \mathrm{GPa}$; visco-plastic reference strain rate $\dot{\varepsilon}_{0_{\mathrm{I}}}=1.0 \mathrm{~s}^{-1}$; visco-plastic exponent $m_{\mathrm{I}}=0.1$; volume fraction $v_{\mathrm{I}}=25 \%$. 


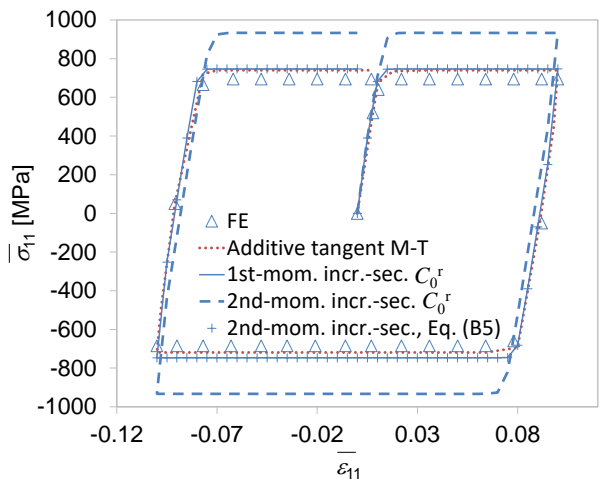

(a) Composites response

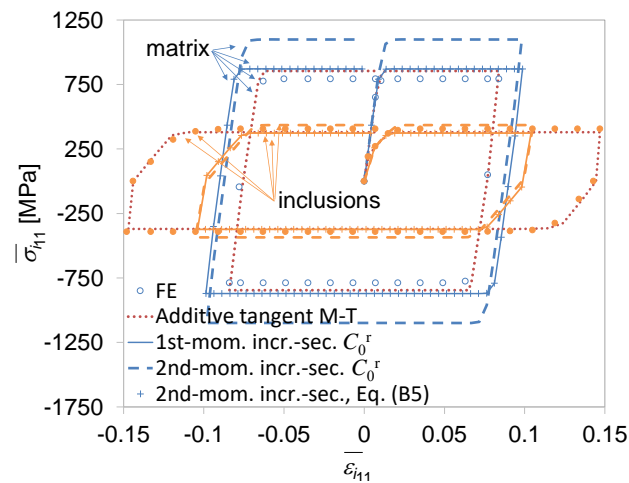

(b) Phases response

Figure B.16: Inelastic solid with soft spherical elasto-viscoplastic inclusions under cyclic loading. The method predictions using the original estimate (43) and the modified estimate (B.2) are compared with the prediction of the additive tangent Mori-Tanaka (M-T) interaction law and the full-field FE results provided by Czarnota et al. (2015).

A uniaxial tension-compression cycle is applied on the composite with a strain amplitude of \pm 0.1 and at a strain rate $\dot{\varepsilon}=0.5 \mathrm{~s}^{-1}$. The incremental-secant MFH predictions using first statistical moment estimates, considering the residual stress in the matrix and the secant operator $C_{0}^{\mathrm{r}}$ since the inclusions are soft, and the incremental-secant MFH predictions with two different assumptions for the second statistical moment estimate of the von Mises stress, i.e. Eq. (43) and Eq. (B.2), are successively considered for this test. The incremental-secant MFH predictions are compared in Fig. B.16 with the direct finite element simulation results and the predictions of additive tangent Mori-Tanaka (M-T) interaction law, both provided by Czarnota et al. (2015). When considering the composite material response in Fig. 16(a), the results obtained with the modified second statistical moment estimate (B.2) correspond to the results obtained with the first statistical moment estimates since the two phases have the same Young's modulus. They are also in agreement with the FE results and the additive tangent Mori-Tanaka (M-T) interaction law predictions. However, when comparing the phases responses in Fig. 16(b), it appears that while the stress levels are predicted in an accurate way when considering the modified second statistical moment estimate (B.2) or the first statistical moment estimates, the strain contrast is underestimated with the incremental-secant MFH predictions.

\section{References}

Aboudi, J., 1996. Micromechanical analysis of composites by the method of cells - update. Applied Mechanics Reviews 49, S83-S91.

Agoras, M., Ponte Castañeda, P., 2013. Iterated linear comparison bounds for viscoplastic porous materials with ellipsoidal microstructures. Journal of the Mechanics and Physics of Solids 61, $701-$ 725 .

Berveiller, M., Zaoui, A., 1978. An extension of the self-consistent scheme to plastically-flowing polycrystals. Journal of the Mechanics and Physics of Solids 26, $325-344$.

Bobeth, M., Diener, G., 1987. Static elastic and thermoelastic field fluctuations in multiphase composites. Journal of the Mechanics and Physics of Solids 35, $137-149$. 
Boudet, J., Auslender, F., Bornert, M., Lapusta, Y., 2016. An incremental variational formulation for the prediction of the effective work-hardening behavior and field statistics of elasto-(visco)plastic composites. International Journal of Solids and Structures 83, 90 - 113.

Brassart, L., Stainier, L., Doghri, I., Delannay, L., 2011. A variational formulation for the incremental homogenization of elasto-plastic composites. Journal of the Mechanics and Physics of Solids 59, 2455 -2475 .

Brassart, L., Stainier, L., Doghri, I., Delannay, L., 2012. Homogenization of elasto-(visco) plastic composites based on an incremental variational principle. International Journal of Plasticity 36, $86-$ 112

Buryachenko, V.A., 2001. Multiparticle Effective Field and Related Methods in Micromechanics of Composite Materials. Applied Mechanics Reviews 54, 1.

Chaboche, J., Kanouté, P., Roos, A., 2005. On the capabilities of mean-field approaches for the description of plasticity in metal matrix composites. International Journal of Plasticity 21, $1409-$ 1434.

Czarnota, C., Kowalczyk-Gajewska, K., Salahouelhadj, A., Martiny, M., Mercier, S., 2015. Modeling of the cyclic behavior of elasticviscoplastic composites by the additive tangent moritanaka approach and validation by finite element calculations. International Journal of Solids and Structures 5657, 96 $-117$.

Doghri, I., Adam, L., Bilger, N., 2010. Mean-field homogenization of elasto-viscoplastic composites based on a general incrementally affine linearization method. International Journal of Plasticity 26, $219-$ 238.

Doghri, I., Brassart, L., Adam, L., Gérard, J.S., 2011. A second-moment incremental formulation for the mean-field homogenization of elasto-plastic composites. International Journal of Plasticity 27, $352-$ 371.

Doghri, I., Ouaar, A., 2003. Homogenization of two-phase elasto-plastic composite materials and structures: Study of tangent operators, cyclic plasticity and numerical algorithms. International Journal of Solids and Structures 40, $1681-1712$.

Doghri, I., Tinel, L., 2005. Micromechanical modeling and computation of elasto-plastic materials reinforced with distributed-orientation fibers. International Journal of Plasticity 21, 1919 - 1940.

Dvorak, G., Wafa, A., Bahei-El-Din, Y., 1994. Implementation of the transformation field analysis for inelastic composite materials. Computational Mechanics 14, 201-228.

Eshelby, J.D., 1957. The determination of the elastic field of an ellipsoidal inclusion, and related problems. Proceedings of the Royal Society of London. Series A, Mathematical and Physical Sciences 241, pp. 376-396.

Feyel, F., 1999. Multiscale $\{$ FE2 $\}$ elastoviscoplastic analysis of composite structures. Computational Materials Science 16, $344-354$.

Geers, M., Kouznetsova, V., Brekelmans, W., 2010. Multi-scale computational homogenization: Trends and challenges. Journal of Computational and Applied Mathematics 234, 2175 - 2182. Fourth International Conference on Advanced \{COmputational\} Methods in \{ENgineering (ACOMEN 2008)

Ghosh, S., Lee, K., Moorthy, S., 1995. Multiple scale analysis of heterogeneous elastic structures using homogenization theory and voronoi cell finite element method. International Journal of Solids and Structures 32, $27-62$

Hill, R., 1965a. Continuum micro-mechanics of elastoplastic polycrystals. Journal of the Mechanics and Physics of Solids $13,89-101$.

Hill, R., 1965b. A self-consistent mechanics of composite materials. Journal of the Mechanics and Physics of Solids 13, $213-222$.

Idiart, M.I., Lahellec, N., 2016. Estimates for the overall linear properties of pointwise heterogeneous solids with application to elasto-viscoplasticity. Journal of the Mechanics and Physics of Solids 97 317 - 332. SI:Pierre Suquet Symposium.

Kanouté, P., Boso, D., Chaboche, J., Schrefler, B., 2009. Multiscale methods for composites: A review. Archives of Computational Methods in Engineering 16, 31-75.

Kouznetsova, V., Brekelmans, W.A.M., Baaijens, F.P.T., 2001. An approach to micro-macro modeling of heterogeneous materials. Computational Mechanics 27, 37-48.

Kröner, E., 1958. Berechnung der elastischen konstanten des vielkristalls aus den konstanten des einkristalls. Zeitschrift für Physik A Hadrons and Nuclei 151, 504-518. 10.1007/BF01337948.

Lahellec, N., Ponte Castañeda, P., Suquet, P., 2011. Variational estimates for the effective response and field statistics in thermoelastic composites with intra-phase property fluctuations. Proceedings of the Royal Society A: Mathematical, Physical and Engineering Science 467, 2224-2246.

Lahellec, N., Suquet, P., 2007a. On the effective behavior of nonlinear inelastic composites: I. incremental 
variational principles. Journal of the Mechanics and Physics of Solids 55, 1932 - 1963.

Lahellec, N., Suquet, P., 2007b. On the effective behavior of nonlinear inelastic composites: II. a secondorder procedure. Journal of the Mechanics and Physics of Solids 55, $1964-1992$.

Lahellec, N., Suquet, P., 2013. Effective response and field statistics in elasto-plastic and elastoviscoplastic composites under radial and non-radial loadings. International Journal of Plasticity

Li, J., Weng, G., 1998. A unified approach from elasticity to viscoelasticity to viscoplasticity of particlereinforced solids. International Journal of Plasticity 14, $193-208$.

LLorca, J., González, C., Molina-Aldareguía, J.M., Segurado, J., Seltzer, R., Sket, F., Rodríguez, M., Sádaba, S., Muñoz, R., Canal, L.P., 2011. Multiscale modeling of composite materials: a roadmap towards virtual testing. Advanced Materials 23, 5130-5147.

Masson, R., Bornert, M., Suquet, P., Zaoui, A., 2000. An affine formulation for the prediction of the effective properties of nonlinear composites and polycrystals. Journal of The Mechanics and Physics of Solids 48, 1203-1227.

Masson, R., Zaoui, A., 1999. Self-consistent estimates for the rate-dependent elastoplastic behaviour of polycrystalline materials. Journal of the Mechanics and Physics of Solids 47, $1543-1568$.

Mercier, S., Molinari, A., 2009. Homogenization of elasticviscoplastic heterogeneous materials: Selfconsistent and mori-tanaka schemes. International Journal of Plasticity 25, 1024 - 1048.

Mercier, S., Molinari, A., Berbenni, S., Berveiller, M., 2012. Comparison of different homogenization approaches for elasticviscoplastic materials. Modelling and Simulation in Materials Science and Engineering 20, 024004 .

Michel, J., Moulinec, H., Suquet, P., 1999. Effective properties of composite materials with periodic microstructure: a computational approach. Computer Methods in Applied Mechanics and Engineering $172,109-143$.

Michel, J., Suquet, P., 2003. Nonuniform transformation field analysis. International Journal of Solids and Structures 40, 6937 - 6955. Special issue in Honor of George J. Dvorak.

Michel, J.C., Moulinec, H., Suquet, P., 2001. A computational scheme for linear and non-linear composites with arbitrary phase contrast. International Journal for Numerical Methods in Engineering 52, $139-160$.

Miehe, C., 2002. Strain-driven homogenization of inelastic microstructures and composites based on an incremental variational formulation. International Journal for Numerical Methods in Engineering 55, $1285-1322$.

Molinari, A., Canova, G., Ahzi, S., 1987. A self consistent approach of the large deformation polycrystal viscoplasticity. Acta Metallurgica 35, 2983-2994.

Molinari, A., El Houdaigui, F., Tóth, L., 2004. Validation of the tangent formulation for the solution of the non-linear eshelby inclusion problem. International Journal of Plasticity 20, $291-307$.

Mori, T., Tanaka, K., 1973. Average stress in matrix and average elastic energy of materials with misfitting inclusions. Acta Metallurgica 21, 571-574. Cited By (since 1996) 1814.

Moulinec, H., Suquet, P., 1995. A fft-based numerical method for computing the mechanical properties of composites from images of their microstructures, in: Pyrz, R. (Ed.), IUTAM Symposium on Microstructure-Property Interactions in Composite Materials. Springer Netherlands. volume 37 of Solid Mechanics and Its Applications, pp. 235-246.

Moulinec, H., Suquet, P., 1998. A numerical method for computing the overall response of nonlinear composites with complex microstructure. Computer Methods in Applied Mechanics and Engineering $157,69-94$

Moulinec, H., Suquet, P., 2003. Intraphase strain heterogeneity in nonlinear composites: a computational approach. European Journal of Mechanics - A/Solids 22, $751-770$.

Nemat-Nasser, S., Hori, M., 2013. Micromechanics: overall properties of heterogeneous materials. volume 37. Elsevier.

Noels, L., Wu, L., Adam, L., 2016. Review of homogenization methods for heterogeneous materials, in Ulrich, P., Schmitz, G.J. (Eds.), Handbook of Software Solutions for ICME. Wiley-VCH, Weinheim, Germany. chapter 6.1.1., pp. 433-441.

Ostlund, R., Golling, S., Oldenburg, M., 2016. Microstructure based modeling of ductile fracture initiation in press-hardened sheet metal structures. Computer Methods in Applied Mechanics and Engineering 302, $90-108$.

Pettermann, H.E., Plankensteiner, A.F., Böhm, H.J., Rammerstorfer, F.G., 1999. A thermo-elastoplastic constitutive law for inhomogeneous materials based on an incremental mori-tanaka approach. Computers \& Structures 71, $197-214$.

Pierard, O., 2006. Micromechanics of inclusion-reinforced composites in elastoplasticity and elasto- 
viscoplasticity : modeling and computation. Ph.D. thesis. Université Catholique de Louvain, LouvainLa-Neuve (Belgium).

Pierard, O., Doghri, I., 2006a. An enhanced affine formulation and the corresponding numerical algorithms for the mean-field homogenization of elasto-viscoplastic composites. International Journal of Plasticity 22, $131-157$.

Pierard, O., Doghri, I., 2006b. Study of various estimates of the macroscopic tangent operator in the incremental homogenization of elastoplastic composites. International Journal for Multiscale Computational Engineering 4, 521-543.

Pierard, O., LLorca, J., Segurado, J., Doghri, I., 2007. Micromechanics of particle-reinforced elastoviscoplastic composites: Finite element simulations versus affine homogenization. International Journal of Plasticity 23, $1041-1060$.

Ponte Castañeda, P., 1991. The effective mechanical properties of nonlinear isotropic composites. Journal of the Mechanics and Physics of Solids 39, 45-71.

Ponte Castañeda, P., 1992. A new variational principle and its application to nonlinear heterogeneous systems. SIAM Journal on Applied Mathematics 52, 1321-1341.

Ponte Castañeda, P., 1996. Exact second-order estimates for the effective mechanical properties of nonlinear composite materials. Journal of the Mechanics and Physics of Solids 44, $827-862$.

Ponte Castañeda, P., 2002a. Second-order homogenization estimates for nonlinear composites incorporating field fluctuations: I - theory. Journal of the Mechanics and Physics of Solids 50, 737 757.

Ponte Castañeda, P., 2002b. Second-order homogenization estimates for nonlinear composites incorporating field fluctuations: II - applications. Journal of the Mechanics and Physics of Solids 50, $759-$ 782.

Ponte Castañeda, P., 2012. Bounds for nonlinear composites via iterated homogenization. Journal of the Mechanics and Physics of Solids 60, $1583-1604$.

Suquet, P., 1995. Overall properties of nonlinear composites: A modified secant moduli theory and its link with ponte castañeda's nonlinear variational procedure. Comptes Rendus de l" Académie des Sciences 320, 563-571.

Talbot, D., Willis, J., 1992. Some simple explicit bounds for the overall behaviour of nonlinear composites. International Journal of Solids and Structures 29, $1981-1987$.

Talbot, D.R.S., Willis, J.R., 1985. Variational principles for inhomogeneous non-linear media. IMA Journal of Applied Mathematics 35, 39-54.

Talbot, D.R.S., Willis, J.R., 1987. Bounds and self-consistent estimates for the overall properties of nonlinear composites. IMA Journal of Applied Mathematics 39, 215-240.

Terada, K., Hori, M., Kyoya, T., Kikuchi, N., 2000. Simulation of the multi-scale convergence in computational homogenization approaches. International Journal of Solids and Structures 37, 22852311.

Wu, L., Doghri, I., Noels, L., 2015a. An incremental-secant mean-field homogenization method with second statistical moments for elasto-plastic composite materials. Philosophical Magazine 95, 33483384. http://dx.doi.org/10.1080/14786435.2015.1087653.

Wu, L., Noels, L., Adam, L., Doghri, I., 2012. Multiscale mean-field homogenization method for fiberreinforced composites with gradient-enhanced damage model. Computer Methods in Applied Mechanics and Engineering 233-236, 164-179.

Wu, L., Noels, L., Adam, L., Doghri, I., 2013a. A combined incremental-secant mean-field homogenization scheme with per-phase residual strains for elasto-plastic composites. International Journal of Plasticity 51, 80-102.

Wu, L., Noels, L., Adam, L., Doghri, I., 2013b. An implicit-gradient-enhanced incremental-secant meanfield homogenization scheme for elasto-plastic composites with damage. International Journal of Solids and Structures 50, $3843-3860$.

Wu, L., Sket, F., Molina-Aldareguia, J., Makradi, A., Adam, L., Doghri, I., Noels, L., 2015b. A study of composite laminates failure using an anisotropic gradient-enhanced damage mean-field homogenization model. Composite Structures 126, $246-264$. 